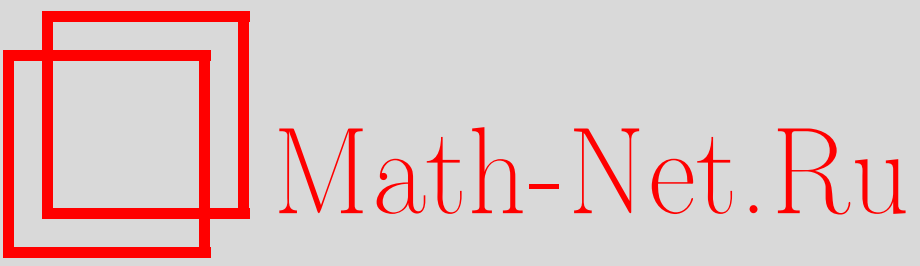

G. Peccati, J.-R. Pycke, Decompositions of stochastic processes based on irreducible group representations, Теория вероятн. и ее примен., 2009, том 54, выпуск 2, 304336

DOI: https://doi.org/10.4213/tvp2704

Использование Общероссийского математического портала Math-Net.Ru подразумевает, что вы прочитали и согласны с пользовательским соглашением http://www . mathnet.ru/rus/agreement

Параметры загрузки:

IP: 52.6 .47 .48

26 апреля 2023 г., 14:54:14 


\title{
DECOMPOSITIONS OF STOCHASTIC PROCESSES BASED ON IRREDUCIBLE GROUP REPRESENTATIONS
}

\begin{abstract}
Пусть $G$ - компактная топологическая группа, действующая на некотором пространстве $Y$. Мы изучаем разложение $Y$-индексированных случайных процессов, основанное на соотношениях ортогональности для характеров неприводимых представлений группы $G$. В частном случае гауссовского процесса с $G$-инвариантным законом такое разложение дает весьма общее истолкование одного классического тождества по распределению (между квадратичными функционалами от броуновского моста), установленного Ватсоном (1961). Кроме того, обсуждается связь с разложениями Карунена-Лоэва и приводятся применения и обобщения, в частности связанные с гауссовскими процессами на торе.
\end{abstract}

Ключевые слова и фразы: случайные процессы, компактные топологические группы, неприводимые представления, квадратичные функционалы, тождество Ватсона, двойные интегралы Винера-Ито, разложения Карунена-Лоэва.

1. Introduction. This paper deals with the study of a class of decompositions for stochastic processes, based on the theory of group representations. As clarified below, the initial impetus for our investigation was provided by the following duplication identity due to Watson (see [24]): if $b$ is a standard Brownian bridge on $[0,1]$, from 0 to 0 , then

$$
\int_{0}^{1}\left(b(s)-\int_{0}^{1} b(u) d u\right)^{2} d s \stackrel{\text { law }}{=} \frac{1}{4}\left\{\int_{0}^{1} b(s)^{2} d s+\int_{0}^{1} b_{*}(s)^{2} d s\right\}
$$

where $b_{*}$ is an independent copy of $b$. The reader is referred to [21, p. 220] for a proof of (1) using Karhunen-Loève expansions, and to [20] for a probabilistic discussion based on several identities in law between Brownian functionals. Recently (see [14]), the second author gave a very short proof of (1),

*Equipe Modal'X, Université Paris Ouest Nanterre La Défense; Laboratoire de Statistiques Théoriques et Appliquées, Université Paris VI, France; e-mail: giovanni.peccati@gmail.com

** Department of Mathematics, University of Évry, France; e-mail: jrpycke@maths.univevry.fr 
suggesting that Watson's result hides indeed a simple algebraic structure. In what follows, we shall bring this structure into light, by showing that (1) is an instance of a quite general phenomenon, related to the invariance properties of the law of $b$. In particular, we will provide an exhaustive answer to a question raised by Z. Shi and M. Yor in [20]: what is the probabilistic interpretation of the factor $1 / 4$ in (1)? Our general framework is roughly as follows.

Let $G$ be a topological compact group acting on a set $Y$, and let $Z(\omega, y)=Z(y)$ be a stochastic process indexed by the elements of $Y$. We will consider a decomposition of the paths of $Z$, realized by means of the orthogonality relations between the characters of the irreducible representations of $G$ (see [7] or [19] for any unexplained notion about representations). More specifically, we define $L^{2}(G)$ to be the space of complex-valued functions on $G$ that are square integrable with respect to the Haar measure, and we denote by $\widehat{G}$ the dual of $G$ (i.e., $\widehat{G}$ is the collection of the equivalence classes of the irreducible representations of $G$ ). Then, a classic result of representation theory states that $L^{2}(G)$ can be decomposed into an orthogonal sum of finite dimensional spaces, indexed by the elements $[\pi]$ of $\widehat{G}$ and known as the spaces of matrix coefficients of irreducible representations. The projection operators on such orthogonal spaces have the form of convolutions with respect to the corresponding characters. Now write $(g, y) \mapsto g \cdot y$ to indicate the action of $G$ on $Y$, and consider a stochastic process $Z(y)$, $y \in Y$, such that, for every fixed $y \in Y$, the application $g \mapsto Z(g \cdot y)$ is in $L^{2}(G)$. One of our main results states that, if the law of $Z$ is invariant with respect to the action of $G$, then the above-described decomposition of $L^{2}(G)$ translates into a (unique) decomposition of $Z$ into the sum of simpler stochastic processes, each indexed by a distinct element of $\widehat{G}$. We write $Z=\sum_{[\pi] \in \widehat{G}} Z^{\pi}$ for such a decomposition. In Section 3 , we prove that, if $Z$ has a $G$-invariant law, then, for distinct $[\pi],[\sigma] \in \widehat{G}$, the processes $Z^{\pi}$ and $Z^{\sigma}$ are noncorrelated (in a probabilistic sense), and such that their paths are orthogonal with respect to any $G$-invariant measure on the parameter space $Y$. In particular, when $Z$ is Gaussian and $[\pi]$ and $[\sigma]$ have real characters, $Z^{\pi}$ and $Z^{\sigma}$ are also Gaussian, and therefore stochastically independent. In the last section we discuss some connections between our decomposition and the Karhunen-Loève expansion (see, for instance, [1]) of suitably regular Gaussian processes.

As a by-product of our analysis, we show that (1) derives from a very particular case of the decomposition described above. In particular, our results will make clear that there are two crucial elements behind (1), namely: (i) since $b_{t} \stackrel{\text { law }}{=} b_{1-t}$ (as stochastic processes), the law of $b(\cdot)-\int_{0}^{1} b(u) d u$ is invariant with respect to the elementary action, of $G=\{1, g\} \simeq \mathbf{Z} / 2 \mathbf{Z}$ on $[0,1]$, given by $1 \cdot t=t$ and $g \cdot t=1-t$, and (ii) Lebesgue measure is invariant 
with respect to the same action of $G$. It follows that the above-described theory can be applied, and (1) turns out to be the result of an orthogonal decomposition of the paths of $b(\cdot)-\int_{0}^{1} b(u) d u$ into two independent components. More to the point, the factor $1 / 4$ on the right-hand side of (1) will appear as the square of a normalization factor $\left(1 / 4=1 /|G|^{2}\right)$, which enters quite naturally into the expression of the projection operators associated to matrix coefficients. The generalizations of (1) given in [13] have similar interpretations in terms of group representations (see Section 4 below for the analysis of a quadruplication identity).

Note that, although we are mainly motivated by finite groups, in the first part of the paper we will work in the general framework of topological compact groups. See also [10]-[12] for some related results concerning processes defined on the sphere or on a compact commutative group.

The paper is organized as follows. In Section 2 we recall some basic facts about group representations and related orthogonality relations. Section 3 deals with decompositions of stochastic processes in the general case. Section 4 studies the specific setup of Gaussian processes, and contains an extended discussion of generalized Volterra processes. In particular, we establish some necessary and sufficient conditions (based on the method of cumulants) to have that such processes verify a relation analogous to (1). In the last section, we prove several refinements and applications, mainly related to Karhunen-Loève expansions and to Gaussian processes indexed by a torus.

\section{Preliminaries and main results from group representation} theory.

2.1. Representations of compact groups and orthogonal decompositions. In this section, we present several definitions and results from the theory of representations of topological compact groups. Our use of this theory is mainly inspired by the discussion contained in [7, Chap. IV], where a strong accent is placed on the so-called Peter-Weyl theorem (see [7, Theorem 4.6.1], as well as the discussion below) and its consequences in terms of the decomposition of the space $L^{2}$ associated with a topological compact group (when endowed with its Haar measure). The reader is referred to [7] for any unexplained definition or result. Other classic references for group representations are the monographs [19] and [9].

A topological group is a pair $(G, \mathbb{G})$, where $G$ is a group and $\mathbb{G}$ is a topology such that the following three conditions are satisfied: (i) $G$ is a Hausdorff topological space, (ii) the multiplication $G \times G \mapsto G:(g, h) \mapsto$ $g h$ is continuous, (iii) the inversion $G \mapsto G: g \mapsto g^{-1}$ is continuous. In what follows, when no further specification is given, $G$ will always denote a topological group (the topology $\mathbb{G}$ being implicitly defined) which is also compact (see, e.g., [6, p. 34]) and such that $\mathbb{G}$ has a countable basis. For such 
a $G$, we denote by $C(G)$ the class of continuous, complex-valued functions on $G ; \mathscr{G}$ is the (Borel) $\sigma$-field generated by $\mathbb{G}$. An immediate consequence (see [7, Section 10.3]) of the structure imposed on $G$, is that $G$ always carries a (unique) positive Borel measure, denoted by $d g$ and known as the Haar measure, such that $\int_{G} d g=1$, and for all $f \in C(G)$ and for all $h \in G$

$$
\begin{aligned}
\int_{G} f(g) d g & =\int_{G} f\left(g^{-1}\right) d g \\
\int_{G} f(h g) d g & =\int_{G} f(g h) d g=\int_{G} f(g) d g \quad \text { (left and right invariance); }
\end{aligned}
$$

we denote by $L^{2}(G, d g)=L^{2}(G)$ the Hilbert space of complex-valued functions on $G$ that are square integrable with respect to $d g$, endowed with the usual inner product $\left\langle f_{1}, f_{2}\right\rangle_{G}=\int_{G} f_{1}(g) \overline{f_{2}(g)} d g$. We denote by $\|\cdot\|_{G}$ the norm associated with $\langle\cdot, \cdot\rangle_{G}$, and we observe that $L^{2}(G)$ is the completion of $C(G)$ with respect to $\|\cdot\|_{G}$.

$\mathrm{R}$ e $\mathrm{m}$ a $\mathrm{rk}$. If $G$ is finite, then $\mathbb{G}$ is necessarily the discrete topology, and $d g$ coincides with the normalized counting measure associated with $G$, that is,

$$
d g=\frac{1}{|G|} \sum_{h \in G} \delta_{h}(d g),
$$

where $\delta_{h}(\cdot)$ stands for the Dirac mass concentrated at $h$, and $|G|$ is the cardinality of $G$.

Let $V$ be a topological vector space over C. A representation of $G$ in $V$ is an homomorphism $\pi$, from $G$ into $\mathbf{G L}(V)$ (the set of complex isomorphisms of $V$ into itself), such that the mapping $G \times V \mapsto V:(g, v) \mapsto \pi(g)(v)$ is continuous. The dimension $d_{\pi}$ of a representation $\pi$ is defined to be the dimension of $V$. A representation $\pi$ of $G$ in $V$ is irreducible, if the only closed $\pi(G)$-invariant subspaces of $V$ are $\{0\}$ and $V$. It is well known that irreducible representations are defined up to equivalence classes (see [7, p. 210]). Following [7], we denote by $[\pi]$ the equivalence class of a given irreducible representation $\pi$; the set of equivalence classes of irreducible representations of $G$ is denoted by $\widehat{G}$, and it is called the dual of $G$. Note that, in our setting, irreducible representations are always finite dimensional. Moreover, we will systematically assume (without loss of generality, see [7, Corollary 4.2.2]) that every irreducible representation is also unitary. Finally, we recall that, according, e.g., to [7, Theorem 4.3.4 (v)], since $G$ is second countable (and therefore metrizable) $\widehat{G}$ is necessarily countable.

To every finite dimensional representation $\pi: G \mapsto \mathbf{G L}(V)$ we associate the mapping

$$
\chi_{\pi}: G \rightarrow \mathbf{C}: g \mapsto \text { Trace } \pi(g),
$$

called the character of $\pi$. Two finite-dimensional representations are equivalent if and only if they have the same character. Moreover, it is easily seen 
that characters are central (that is, for every $x, g \in G, \chi_{\pi}\left(x^{-1} g x\right)=\chi_{\pi}(g)$ ) and continuous functions on $G$.

In this paper, we develop some Hilbert space techniques that are directly based on the orthogonality relations between the characters of distinct irreducible representations. To this end, with every $[\pi] \in \widehat{G}$ we associate a finite dimensional subspace $M_{\pi} \subseteq L^{2}(G)$ in the following way. Select an element $\pi: G \mapsto \mathbf{G L}(V)$ in $[\pi]$, as well as a basis $\mathbf{e}=\left\{\mathbf{e}_{1}, \ldots, \mathbf{e}_{n}\right\}$ of $V$ (plainly, $n=d_{\pi}$ ) with respect to which $\pi$ is unitary; the space $M_{\pi}$ is defined as the set of the (complex) linear combinations of the matrix coefficients associated with $\pi$ and with the basis e, that is, $M_{\pi}$ is composed of the linear combinations of the functions $g \mapsto \pi(g)_{k}^{j}, j, k=1, \ldots, n$, where, for each $g \in G$, $\left\{\pi(g)_{k}^{j}: j, k=1, \ldots, n\right\}$ is the matrix representation of $\pi(g)$ with respect to the basis e. Note that such a definition is well given, since $M_{\pi}$ does not depend on the choices of the representative element of $[\pi]$ and of the basis of $V$. Of course, $M_{\pi}$ is finite dimensional (and therefore closed; more precisely: $\operatorname{dim} M_{\pi}=d_{\pi}^{2}$, see [7, Theorem 4.3.4], as well as the discussion below) and $M_{\pi} \subseteq C(G)$, for every $[\pi] \in \widehat{G}$.

Before stating one of the crucial results for our analysis, we introduce a convolution operation on $L^{2}(G)$, which is defined, for $f, k \in L^{2}(G)$, by the formula

$$
(f * k)(u)=\int_{G} f(g) k\left(g^{-1} u\right) d g=\int_{G} f\left(u g^{-1}\right) k(g) d g, \quad u \in G .
$$

The following result summarizes all the orthogonality relations - associated with the notion of character - that are relevant to our discussion (for proofs and further analysis in this direction, the reader is referred to $[7$, Sections 4.2-4.6]).

Theorem 1. Let the above notation and assumptions prevail. Then,

1) if $[\pi],[\sigma] \in \widehat{G}$, and $[\pi] \neq[\sigma]$, then the spaces $M_{\pi}$ and $M_{\sigma}$ are orthogonal in $L^{2}(G)$;

2) for every $[\pi] \in \widehat{G}$ the orthogonal projection operator, from $L^{2}(G)$ to $M_{\pi}$, is given by

$$
\mathbf{E}_{\pi}: L^{2}(G) \rightarrow M_{\pi}: f \mapsto d_{\pi}\left(f * \chi_{\pi}\right):=\mathbf{E}_{\pi} f ;
$$

3) the class $\left\{M_{\pi}:[\pi] \in \widehat{G}\right\}$ is total in $L^{2}(G)$, and therefore

$$
L^{2}(G)=\bigoplus_{[\pi] \in \widehat{G}} M_{\pi}
$$

where $\bigoplus$ stands for a direct Hilbert space (orthogonal) sum;

4) for every $[\pi] \in \widehat{G}$,

$$
\left\langle\chi_{\pi}, \chi_{\pi}\right\rangle_{G}=1, \quad \mathbf{E}_{\pi} \chi_{\pi}=\chi_{\pi} \quad \text { and } \quad \mathbf{E}_{\sigma} \chi_{\pi}=0 \quad \text { if }[\sigma] \in \widehat{G} \text { and }[\pi] \neq[\sigma],
$$

and consequently $\left\{\chi_{\pi}:[\pi] \in \widehat{G}\right\}$ is an orthonormal system in $L^{2}(G)$. 
$\mathrm{R}$ e $\mathrm{m}$ a r k. (i) According, e.g., to [7, p. 221], the right regular representation $R^{*}$ of $G$ on $L^{2}(G)$ is defined as $\left(R^{*}(g) f\right)(x)=f(x g)$, where $g, x \in G$ and $f \in L^{2}(G)$. Then, for every $[\pi] \in \widehat{G}$, the space $M_{\pi}$ coincides with the $\pi$-isotypical subspace associated with $R^{*}$ (see [7, Theorem 4.4.5]). More to the point (see [7, Corollary 4.3.6]), the restriction of $R^{*}$ on $M_{\pi}$ is equal to the direct sum of $d_{\pi}$ copies of $\pi$.

(ii) Since, for every $[\pi] \in \widehat{G}$, the function $G \ni g \mapsto \chi_{\pi}(g)$ is conjugacyinvariant, the projection $\mathbf{E}_{\pi} f$, as defined in (3), is also equal to $d_{\pi}\left(\chi_{\pi} * f\right)$.

(iii) Point 3 of Theorem 1 can be seen as a direct consequence of the Peter-Weyl theorem (see [7, Theorem 4.6.1]), stating that the characters of the irreducible representations of $G$ form an orthonormal basis of the subspace of $L^{2}(G)$ composed of central and square integrable functions.

(iv) For future reference, we recall that the following four conditions are equivalent (see [7, p. 235]): (a) $G$ is Abelian, (b) $d_{\pi}=1$ for every $[\pi] \in \widehat{G}$, (c) every $f \in C(G)$ is conjugacy-invariant, (d) the convolution operation defined in (2) is commutative. In particular, if $G$ is Abelian, then the system $\left\{\chi_{\pi}:[\pi] \in \widehat{G}\right\}$ is orthonormal and complete in $L^{2}(G)$. If $G$ is Abelian and finite, then $|G|=|\widehat{G}|$.

2.2. Actions and decompositions of complex-valued functions. Consider a measurable space $(X, \mathscr{X})$. In this paper, a left action $A$ of $G$ on $X$ is a $(\mathscr{G} \otimes \mathscr{X})$-measurable function, from $G \times X$ to $X$ (recall that $\mathscr{G}$ is the Borel $\sigma$-field of $G$ ) such that, for every $g, h \in G$ and $x \in X$,

$$
A(g h, x)=A(g, A(h, x)) .
$$

A right action can be defined in a similar way, but we will deal only with left actions; for the sake of simplicity, in the sequel left actions are simply called actions. When there is no ambiguity on the action $A$, we will sometimes use the customary abbreviation

$$
A(g, x)=g \cdot x \quad(g \in G, x \in X) .
$$

A $\sigma$-finite, positive measure $\nu$ on $(X, \mathscr{X})$ is said to be invariant with respect to the action $A$ of $G$ (or simply, again when there is no ambiguity on the action $A, G$-invariant) if, for every complex valued function $f \in L^{1}(\nu)$,

$$
\int_{X} f(x) \nu(d x)=\int_{X} f(A(g, x)) \nu(d x)=\int_{X} f(g \cdot x) \nu(d x), \quad \text { for every } g \in G \text {. }
$$

Now fix an action $A$ of $G$ on $X$, and consider a measurable function $Z: X \rightarrow \mathbf{C}$. We associate to $Z$ the function

$$
Z_{*}: G \times X \rightarrow \mathbf{C}:(g, x) \mapsto Z_{*}(g, x)=Z(A(g, x))=Z(g \cdot x),
$$

which is of course $\mathscr{G} \otimes \mathscr{X}$-measurable. For each fixed $x \in X$, we define the $\mathscr{G}$-measurable function

$$
Z_{G}[x]: G \rightarrow \mathbf{C}: g \mapsto Z_{*}(g, x) ;
$$


analogously, for each fixed $g \in G$, we introduce

$$
Z_{X}[g]: X \rightarrow \mathbf{C}: x \mapsto Z_{*}(g, x),
$$

which defines in turn a $\mathscr{X}$-measurable mapping. If, for some fixed $x \in X$, the above-introduced function $Z_{G}[x]$ is an element of $L^{2}(G)$, we set, for each $[\pi] \in \widehat{G}$ and $g \in G$,

$$
Z_{*}^{\pi}(g, x)=\mathbf{E}_{\pi} Z_{G}[x](g),
$$

where, by using Theorem 1 and (2),

$$
\begin{aligned}
\mathbf{E}_{\pi} Z_{G}[x](g) & =d_{\pi} \int_{G} \chi_{\pi}(h) Z_{G}[x]\left(h^{-1} g\right) d h \\
& =d_{\pi} \int_{G} \chi_{\pi}(h) Z\left(h^{-1} g \cdot x\right) d h, \quad g \in G .
\end{aligned}
$$

As usual, we write $e$ to indicate the identity element of the group $G$. If, for a measurable mapping $Z: X \rightarrow \mathbf{C}, Z_{G}[x] \in L^{2}(G)$ for every $x \in X$, we introduce the two functions, defined, respectively, for a fixed $x \in X$ and for a fixed $g \in G$,

$$
\begin{array}{ll}
Z_{G}^{\pi}[x]: G \rightarrow \mathbf{C :} \quad & g \mapsto Z_{*}^{\pi}(g, x), \\
Z_{X}^{\pi}[g]: X \rightarrow \mathbf{C :} & x \mapsto Z_{*}^{\pi}(g, x) ;
\end{array}
$$

finally, for such a $Z$, we set

$$
\begin{aligned}
Z^{\pi}(x) & =Z_{X}^{\pi}[e](x)=Z_{*}^{\pi}(e, x)=\mathbf{E}_{\pi} Z_{G}[x](e) \\
& =d_{\pi} \int_{G} \chi_{\pi}(g) Z\left(g^{-1} \cdot x\right) d g, \quad x \in X .
\end{aligned}
$$

Note that, since $A$ is a left action,

$$
Z_{X}^{\pi}[g](x)=Z^{\pi}(g \cdot x), \quad g \in G, \quad x \in X .
$$

$\mathrm{R}$ e $\mathrm{m}$ a r k. If the function $Z$ is such that $Z_{G}[x] \in L^{2}(G)$ for every $x \in X$, then the mapping $(g, x) \mapsto Z_{*}^{\pi}(g, x)$ is $(\mathscr{G} \otimes \mathscr{X})$-measurable. It follows that the two mappings $Z_{G}^{\pi}[x]$ and $Z_{X}^{\pi}[g]$ defined in (11) are, respectively, $\mathscr{G}$-measurable and $\mathscr{X}$-measurable. In particular, the application $x \mapsto Z^{\pi}(x)$ (as defined in (12)) is a $\mathscr{X}$-measurable mapping.

The following result turns out to be the key tool of our analysis.

Theorem 2. Under the above notation and assumptions, fix an action $A$ of the group $G$ on $X$. Consider moreover two measurable functions $S, Z: X \rightarrow \mathbf{C}$ such that for each $x \in X$, the functions $S_{G}[x], Z_{G}[x]$ are in $L^{2}(G)$. Then,

1) for any $[\pi],[\sigma] \in \widehat{G}$ such that $[\pi] \neq[\sigma]$, and for arbitrary points $x_{1}, x_{2} \in X$, the following orthogonality relation is satisfied:

$$
\left\langle S_{G}^{\pi}\left[x_{1}\right], Z_{G}^{\sigma}\left[x_{2}\right]\right\rangle_{G}=0
$$


2) for every $x \in X$,

$$
Z_{G}[x]=\sum_{[\pi] \in \widehat{G}} Z_{G}^{\pi}[x] \quad \text { and } \quad S_{G}[x]=\sum_{[\pi] \in \widehat{G}} S_{G}^{\pi}[x]
$$

where the convergence of the (possibly infinite) series takes place in $L^{2}(G)$, and for any $x_{1}, x_{2} \in X$

$$
\left\langle S_{G}\left[x_{1}\right], Z_{G}\left[x_{2}\right]\right\rangle_{G}=\sum_{[\pi] \in \widehat{G}}\left\langle S_{G}^{\pi}\left[x_{1}\right], Z_{G}^{\pi}\left[x_{2}\right]\right\rangle_{G},
$$

with convergence in $\ell^{2}$;

3) in addition to the previous assumptions, suppose there exists a $G$ invariant measure $\nu$ on $(X, \mathscr{X})$ such that the functions $Z_{*}$ and $S_{*}$, defined according to (6), are elements of

$$
L^{2}(G \times X, \mathscr{G} \otimes \mathscr{X}, d g \times \nu(d x)):=L^{2}(d g \times \nu(d x))
$$

and also, for every $g \in G, Z_{X}[g], S_{X}[g] \in L^{2}(X, \mathscr{X}, \nu(d x)):=L^{2}(\nu(d x))$; then, for every $[\pi] \in \widehat{G}$ and every $g \in G$, we have $Z_{*}^{\pi}, S_{*}^{\pi} \in L^{2}(d g \times \nu(d x))$, $Z_{X}^{\pi}[g], S_{X}^{\pi}[g] \in L^{2}(\nu(d x))$, and moreover

$$
\int_{X} S_{X}^{\pi}[g](x) \overline{Z_{X}^{\sigma}[g](x)} \nu(d x)=0
$$

for every $[\pi],[\sigma] \in \widehat{G}$ such that $[\pi] \neq[\sigma]$;

4) under the assumptions and notation of point 3$)$,

$$
Z_{*}=\sum_{[\pi] \in \widehat{G}} Z_{*}^{\pi} \quad \text { and } \quad S_{*}=\sum_{[\pi] \in \widehat{G}} S_{*}^{\pi}
$$

where the series are orthogonal and convergent in $L^{2}(d g \times \nu(d x))$, and therefore

$$
\left\langle Z_{*}, S_{*}\right\rangle_{L^{2}(d g \times \nu(d x))}=\sum_{[\pi] \in \widehat{G}}\left\langle Z_{*}^{\pi}, S_{*}^{\pi}\right\rangle_{L^{2}(d g \times \nu(d x))} ;
$$

5) under the assumptions and notation of point 3), for every $g \in G$,

$$
Z_{X}[g](x)=\sum_{[\pi] \in \widehat{G}} Z_{X}^{\pi}[g](x) \quad \text { and } \quad S_{X}[g](x)=\sum_{[\pi] \in \widehat{G}} S_{X}^{\pi}[g](x),
$$

where the series are orthogonal and convergent in $L^{2}(\nu(d x))$, and

$$
\left\langle Z_{X}[g], S_{X}[g]\right\rangle_{L^{2}(\nu(d x))}=\sum_{[\pi] \in \widehat{G}}\left\langle Z_{X}^{\pi}[g], S_{X}^{\pi}[g]\right\rangle_{L^{2}(\nu(d x))} .
$$


$\mathrm{R}$ e $\mathrm{m}$ a $\mathrm{r}$ k. When $G$ is finite, $\widehat{G}$ is also finite (since $|\widehat{G}|$ coincides with the number of conjugacy classes in $G$ ). In this case, point 2 ) of Theorem 2 gives a decomposition of the function $Z: X \rightarrow \mathbf{C}$. As a matter of fact, for every $x \in X$,

$$
Z(x)=\sum_{[\pi] \in \widehat{G}} Z^{\pi}(x)
$$

where the sum is finite, and on the right-hand side we use the notation introduced in (12).

P r o of of Theorem 2. 1) By definition, $S_{G}^{\pi}\left[x_{1}\right]$ and $Z_{G}^{\sigma}\left[x_{2}\right]$ equal the orthogonal projections, respectively, of $S_{G}\left[x_{1}\right]$ and $Z_{G}\left[x_{2}\right]$, on the finite dimensional spaces $M_{\pi}$ and $M_{\sigma}$. Since, according to point 1) of Theorem $1, M_{\pi}$ and $M_{\sigma}$ are orthogonal in $L^{2}(G)$, relation (14) follows.

2) Relation (15) is an immediate consequence of (4), whereas (16) is a standard formula of the Parseval-Plancherel type.

3) Observe first that, by assumption, $\int_{X}\left[\int_{G}\left|Z_{*}(h, x)\right|^{2} d h\right] \nu(d x)<+\infty$. Moreover, we observe that, for every $[\pi] \in \widehat{G}$, the continuous function $\left|\chi_{\pi}\right|: G \rightarrow \mathbf{R}_{+}$is bounded by a constant $\alpha_{\pi} \in(0,+\infty)$ (since $G$ is compact), and therefore, thanks to the right invariance of the Haar measure $d h$ and the Jensen inequality,

$$
\begin{aligned}
\int_{X}\left|Z_{X}^{\pi}[g](x)\right|^{2} \nu(d x) & =d_{\pi}^{2} \int_{X}\left|\int_{G} \chi_{\pi}(h) Z_{*}\left(h^{-1} g, x\right) d h\right|^{2} \nu(d x) \\
& \leqslant d_{\pi}^{2} \alpha_{\pi}^{2} \int_{X}\left[\int_{G}\left|Z_{*}\left(h^{-1} g, x\right)\right|^{2} d h\right] \nu(d x) \\
& =d_{\pi}^{2} \alpha_{\pi}^{2} \int_{X}\left[\int_{G}\left|Z_{*}(h, x)\right|^{2} d h\right] \nu(d x)<+\infty
\end{aligned}
$$

Also,

$$
\begin{aligned}
\int_{G} \int_{X}\left|Z_{*}^{\pi}(g, x)\right|^{2} \nu(d x) d g & =\int_{G} \int_{X}\left|Z_{G}^{\pi}[g](x)\right|^{2} \nu(d x) d g \\
& \leqslant d_{\pi}^{2} \alpha_{\pi}^{2} \int_{G} \int_{X}\left[\int_{G}\left|Z_{*}(h, x)\right|^{2} d h\right] \nu(d x) d g \\
& =d_{\pi}^{2} \alpha_{\pi}^{2} \int_{X}\left[\int_{G}\left|Z_{*}(h, x)\right|^{2} d h\right] \nu(d x)<+\infty
\end{aligned}
$$

since $\int_{G} d g=1$. It follows that $Z_{*}^{\pi} \in L^{2}(d g \times \nu(d x))$ and $Z_{X}^{\pi}[g] \in L^{2}(\nu(d x))$ for any $g \in G$, and an analogous conclusion holds for $S$. We may prove (17) by using an easy declination of the «averaging» technique (see, for instance, $[7$, Section 4.2$])$. Indeed, for $[\pi],[\sigma] \in \widehat{G}$ such that $[\pi] \neq[\sigma]$, thanks to formula (13) and the $G$-invariance of $\nu$, 


$$
\begin{aligned}
\int_{X} S_{X}^{\pi}[g](x) \overline{Z_{X}^{\sigma}[g](x)} \nu(d x) & =\int_{X} S^{\pi}(g \cdot x) \overline{Z^{\sigma}(g \cdot x)} \nu(d x) \\
& =\int_{G} \int_{X} S^{\pi}(h \cdot x) \overline{Z^{\sigma}(h \cdot x)} \nu(d x) d h \\
& =\int_{X}\left[\int_{G} S^{\pi}(h \cdot x) \overline{Z^{\sigma}(h \cdot x)} d h\right] \nu(d x) \\
& =\int_{X}\left\langle S_{G}^{\pi}[x], Z_{G}^{\sigma}[x]\right\rangle_{G} \nu(d x)=0,
\end{aligned}
$$

where we have used a standard Fubini theorem, as well as point 1) of Theorem 2.

4) The first part derives immediately from points 1) and 2), as well as the fact that $Z_{*}, S_{*} \in L^{2}(d g \times \nu(d x))$ by assumption. Formula (19) is again of the Parseval-Plancherel type.

5) Formula (20) derives from the elementary relation

$$
\begin{aligned}
\int_{X}\left|Z_{X}[g](x)-\sum_{[\pi] \in \widehat{G}} Z_{X}^{\pi}[g](x)\right|^{2} \nu(d x) \\
\quad=\int_{G} \int_{X}\left|Z_{*}(h, x)-\sum_{[\pi] \in \widehat{G}} Z_{*}^{\pi}(h, x)\right|^{2} \nu(d x) d h=0,
\end{aligned}
$$

where the first equality is due to the $G$-invariance of $\nu$, and the second comes from point 4). Relation (21) is straightforward.

3. Decompositions of stochastic processes: general results. Let $(\Omega, \mathscr{F}, \mathbf{P})$ be a probability space, and let $(Y, \mathscr{Y})$ be a measurable space. A $Y$ indexed stochastic process $Z$ is a $(\mathscr{F} \otimes \mathscr{Y})$-measurable application $Z: \Omega \times Y \rightarrow$ C: $(\omega, y) \mapsto Z(\omega, y)^{1)}$. To simplify some arguments, we shall systematically suppose that the $\sigma$-field $\mathscr{F}$ contains the singletons, that is, $\{\omega\} \in \mathscr{F}$ for every $\omega \in \Omega$.

In this section, the product space $\Omega \times Y$ will play roughly the same role as the space $(X, \mathscr{X})$ in Section 2 . As a consequence, we shall sometimes use the compact notation

$$
\Omega \times Y=X_{0}, \quad \mathscr{F} \otimes \mathscr{Y}=\mathscr{X}_{0},
$$

and write $x_{0}$ to indicate the generic element $(\omega, y)$ of $X_{0}$. Given a topological compact group $G$ and an action $A$ of $G$ on $X_{0}$, for fixed $y \in Y$ and $g \in G$, we write $Z(g \cdot y)$ to indicate the random variable $\Omega \ni \omega \mapsto Z(g \cdot(\omega, y))$.

1) We write $Z(y)$, for fixed $y \in Y$, to indicate the random variable $\omega \mapsto Z(\omega, y)$. 
We say that the law of a family $\mathbf{Z}=\left\{Z_{i}: i \in I\right\}$ of stochastic processes is invariant with respect to the action $A$ of $G$ on $X_{0}$ (or, simply, $G$-invariant) if, for every $n \geqslant 1$ and every continuous, bounded function $f$ on $\mathbf{C}^{n}$,

$$
\mathbf{E}\left[f\left(Z_{i_{1}}\left(y_{1}\right), \ldots, Z_{i_{n}}\left(y_{n}\right)\right)\right]=\mathbf{E}\left[f\left(Z_{i_{1}}\left(g \cdot y_{1}\right), \ldots, Z_{i_{n}}\left(g \cdot y_{n}\right)\right)\right]
$$

for every $g \in G$, every $\left(y_{1}, \ldots, y_{n}\right) \in Y^{n}$, and every $\left(i_{1}, \ldots, i_{n}\right) \in I^{n}$.

$\mathrm{R}$ e m a r k. Every action $A^{\prime}$ of $G$ on $Y$ always defines an action $A$ on $X_{0}$, through the relation: for every $x_{0}=(\omega, y) \in X_{0}$,

$$
A\left(g, x_{0}\right)=g \cdot x_{0}=\left(\omega, A^{\prime}(g, y)\right) .
$$

Analogously, every action $\underline{A}^{\prime}$ of $G$ on $\Omega$ defines an action $\underline{A}$ on $X_{0}$ : for every $x_{0}=(\omega, y) \in X_{0}$,

$$
\underline{A}\left(g, x_{0}\right)=g \cdot x_{0}=\left(\underline{A}^{\prime}(g, \omega), y\right) ;
$$

note that if $A$ has the form (24) and $Y$ is homogeneous, then every $Y$ indexed process $Z$ with a $G$-invariant law is also stationary, in the sense that $Z(y) \stackrel{\text { law }}{=} Z\left(y^{\prime}\right)$ for every $y, y^{\prime} \in Y$.

In the sequel, whenever it is given an action $A^{\prime}:(g, y) \mapsto g \cdot y$ of $G$ on $Y$, we will write $g \cdot x_{0}, x_{0} \in X_{0}$, to indicate the image of the action $A$ on $X_{0}$ defined in (24); a similar convention, based on (25), holds for actions $\underline{A}^{\prime}$ on $\Omega$. Moreover, we will systematically work under the following assumption.

A s s u m ption A. Every $Y$-indexed stochastic process $Z$ considered in the following (not necessarily with a $G$-invariant law) is such that, for every $x_{0}=(\omega, y) \in \Omega \times Y$, the mapping $g \mapsto Z\left(g \cdot x_{0}\right)$ is an element of $L^{2}(G)$.

$\mathrm{R}$ e $\mathrm{m}$ a r k. Assumption A can be relaxed in several directions: for instance, at the cost of some heavier notation, most of the subsequent results can immediately be extended to stochastic processes $Z$ such that, for every fixed $y \in Y$, the mapping $g \mapsto Z(g \cdot y)$ is in $L^{2}(G) \mathbf{P}$-a.s. Note that when $G$ is finite Assumption A becomes immaterial.

Now fix an action $A$ of $G$ on $X_{0}$. To every $Y$-indexed stochastic process $Z$ we associate: the mapping $Z_{*}: G \times X_{0} \mapsto \mathbf{C}$, according to (6), and the mappings $Z_{G}\left[x_{0}\right]: G \mapsto \mathbf{C}$ and $Z_{X_{0}}[g]: X_{0} \mapsto \mathbf{C}$ as given, respectively, by (7) for fixed $x_{0}=(\omega, y) \in X_{0}$, and by (8) for fixed $g \in G$. Analogously, for every $[\pi] \in \widehat{G}$, the mapping $Z_{*}^{\pi}: G \times X_{0} \mapsto \mathbf{C}$ is defined according to (9), whereas, for fixed $x_{0} \in X_{0}$ and for fixed $g \in G$, respectively, $Z_{G}^{\pi}\left[x_{0}\right]: G \mapsto \mathbf{C}$ and $Z_{X_{0}}^{\pi}[g]: X_{0} \mapsto \mathbf{C}$, are defined through (11). Finally, $Z^{\pi}: X_{0} \mapsto \mathbf{C}$ is given by $(12)$.

Proposition 1. Under the above notation and assumptions:

1) for every fixed $x_{0} \in X_{0}$ and for every $[\pi] \in \widehat{G}, Z_{G}\left[x_{0}\right]$ and $Z_{G}^{\pi}\left[x_{0}\right]$ are $(G, \mathscr{G})$-measurable functions;

2) for every fixed $g \in G$ and for every $[\pi] \in \widehat{G}, Z_{X_{0}}[g]$ and $Z_{X_{0}}^{\pi}[g]$ are $Y$-indexed stochastic processes; 
3) if $Z$ has a $G$-invariant law, then the following three statements hold: (3-i) for every $g \in G$, the law of $Z_{X_{0}}[g]$ is $G$-invariant and equal to the law of $Z$; (3-ii) for every $[\pi] \in \widehat{G}$ and $g \in G$, the law of $Z_{X_{0}}^{\pi}[g]$ is $G$-invariant and equal to the law of $Z^{\pi}$; (3-iii) the set of stochastic processes $\left\{Z, Z^{\pi}:[\pi] \in \widehat{G}\right\}$ has a $G$-invariant law.

P r o o f. Points 1) and 2) are straightforward. Point (3-i) can be derived immediately from the relation: for every $x_{0} \in X_{0}$

$$
Z_{X_{0}}[g]\left(h \cdot x_{0}\right)=Z\left(g h \cdot x_{0}\right), \quad \forall h \in G,
$$

and the fact that the law of $Z$ is $G$-invariant. To prove (3-ii), we can first use the invariance properties of $d g$, as well as the fact that $\chi_{\pi}(\cdot)$ is central for every $[\pi] \in \widehat{G}$, to obtain that for any $h \in G$

$$
\begin{aligned}
Z^{\pi}(h \cdot x) & =\int_{G} Z(g h \cdot x) \chi_{\pi}\left(g^{-1}\right) d g=\int_{G} Z(g \cdot x) \chi_{\pi}\left(h g^{-1}\right) d g \\
& =\int_{G} Z(g \cdot x) \chi_{\pi}\left(g^{-1} h\right) d g=\int_{G} Z(h \cdot(g \cdot x)) \chi_{\pi}\left(g^{-1}\right) d g \\
& =\int_{G} Z_{X}[h](g \cdot x) \chi_{\pi}\left(g^{-1}\right) d g,
\end{aligned}
$$

from which we deduce that $Z^{\pi}$ has a $G$-invariant law since, thanks to (3-i), $Z_{X}[h]$ has the same law as $Z$. To conclude just use (13) and again (3-i) applied to the process $Z^{\pi}$. Point (3-iii) derives immediately from (26). Proposition 1 is proved.

The following result translates the first part of Theorem 2 into the context of this section. It shows, in particular, that any $G$-invariant stochastic process admits a pointwise $L^{2}$-decomposition in terms of simpler $G$-invariant stochastic processes, indexed by the elements of $\widehat{G}$.

Theorem 3. Let the above notation prevail, and consider an action $A$ of $G$ on $X_{0}=\Omega \times Y$. Let $S$ and $Z$ be two $Y$-indexed stochastic processes verifying Assumption $\mathrm{A}$, and fix $[\pi],[\sigma] \in \widehat{G}$ such that $[\pi] \neq[\sigma]$. Then,

1) for any $\left(\omega_{1}, y_{1}\right),\left(\omega_{2}, y_{2}\right) \in X_{0},\left\langle S_{G}^{\pi}\left[\left(\omega_{1}, y_{1}\right)\right], Z_{G}^{\sigma}\left[\left(\omega_{2}, y_{2}\right)\right]\right\rangle_{G}=0$; $L^{2}(\mathbf{P})$

2) if, for some $y_{1}, y_{2} \in Y, S\left(y_{1}\right), Z\left(y_{2}\right) \in L^{2}(\mathbf{P})$, then $S^{\pi}\left(y_{1}\right), Z^{\sigma}\left(y_{2}\right) \in$

3) if the vector $(S, Z)$ has a $G$-invariant law and $S\left(y_{1}\right), Z\left(y_{2}\right) \in L^{2}(\mathbf{P})$, then

$$
\mathbf{E}\left[S^{\pi}\left(y_{1}\right) \overline{Z^{\sigma}\left(y_{2}\right)}\right]=0 ;
$$

4) if $S$ has a $G$-invariant law and $S\left(y_{1}\right) \in L^{2}(\mathbf{P})$, then

$$
S\left(y_{1}\right)=\sum_{[\pi] \in \widehat{G}} S^{\pi}\left(y_{1}\right),
$$

where the series on the right-hand side is orthogonal and convergent in $L^{2}(\mathbf{P})$. 
P r o o f. Point 1) is a direct consequence of point 1) of Theorem 2, whereas point 2) derives from the inequality

$$
\begin{aligned}
\mathbf{E}\left|S^{\pi}\left(y_{1}\right)\right|^{2} & =d_{\pi}^{2} \int_{\Omega} \mathbf{P}(d \omega)\left|\int_{G} \chi_{\pi}(g) S\left(g^{-1} \cdot\left(\omega, y_{1}\right)\right) d g\right|^{2} \\
& \leqslant d_{\pi}^{2} \alpha_{\pi}^{2} \int_{\Omega} \mathbf{P}(d \omega) \int_{G}\left|S\left(g^{-1} \cdot\left(\omega, y_{1}\right)\right)\right|^{2} d g \\
& =d_{\pi}^{2} \alpha_{\pi}^{2} \int_{G} \mathbf{E}\left|S\left(g^{-1} \cdot y_{1}\right)\right|^{2} d g=d_{\pi}^{2} \alpha_{\pi}^{2} \mathbf{E}\left|S\left(y_{1}\right)\right|^{2}<+\infty,
\end{aligned}
$$

and a similar calculation for $Z^{\pi}$. To see point 3 ), just write, due to the $G$-invariance of $(S, Z)$ and the fact that $\int_{G} d g=1$,

$$
\begin{aligned}
\mathbf{E}\left[S^{\pi}\left(y_{1}\right) \overline{Z^{\sigma}\left(y_{2}\right)}\right] & =\int_{G} \mathbf{E}\left[S^{\pi}\left(g \cdot y_{1}\right) \overline{Z^{\sigma}\left(g \cdot y_{2}\right)}\right] d g \\
& =\int_{\Omega}\left\{\int_{G} S^{\pi}\left[\left(\omega, y_{1}\right)\right](g) \overline{Z^{\sigma}\left[\left(\omega, y_{2}\right)\right](g)} d g\right\} \mathbf{P}(d \omega) \\
& =\int_{\Omega}\left\langle S_{G}^{\pi}\left[\left(\omega, y_{1}\right)\right], Z_{G}^{\sigma}\left[\left(\omega, y_{2}\right)\right]\right\rangle_{G} \mathbf{P}(d \omega)=0,
\end{aligned}
$$

where we have used a Fubini theorem, as well as point 1), with $\omega_{1}=\omega_{2}=\omega$. To prove point 4$)$, let $[\pi(i)], i=1,2, \ldots$, be an enumeration of the elements of $\widehat{G}$, and observe that, according to (15), for every $x_{0}=\left(\omega, y_{1}\right) \in X_{0}$

$$
\lim _{N \rightarrow+\infty} \int_{G}\left|S_{G}\left[x_{0}\right](g)-\sum_{i=1}^{N} S_{G}^{\pi(i)}\left[x_{0}\right](g)\right|^{2} d g=0,
$$

and also, thanks to (16),

$$
\begin{aligned}
\int_{G}\left|S_{G}\left[x_{0}\right](g)-\sum_{i=1}^{N} S_{G}^{\pi(i)}\left[x_{0}\right](g)\right|^{2} d g & =\int_{G}\left|\sum_{i=N+1}^{\infty} S_{G}^{\pi(i)}\left[x_{0}\right](g)\right|^{2} d g \\
& \leqslant \int_{G}\left|S_{G}\left[x_{0}\right](g)\right|^{2} d g .
\end{aligned}
$$

Now observe that, for fixed $y_{1} \in Y$, the random variable $\omega \mapsto \int_{G} \mid S(g$. $\left.\left(\omega, y_{1}\right)\right)\left.\right|^{2} d g$ is in $L^{1}(\mathbf{P})$, since, due to the $G$-invariance of the law of $S$,

$$
\int_{\Omega}\left[\int_{G}\left|S\left(g \cdot\left(\omega, y_{1}\right)\right)\right|^{2} d g\right] \mathbf{P}(d \omega)=\int_{G} \mathbf{E}\left|S\left(g \cdot y_{1}\right)\right|^{2} d g=\mathbf{E}\left|S\left(y_{1}\right)\right|^{2}<+\infty .
$$

Finally, according to point (3-iii) of Proposition 1, the class $\left\{S, S^{\pi}:[\pi] \in \widehat{G}\right\}$ has a $G$-invariant law, and therefore

$$
\begin{aligned}
\mathbf{E}\left|S\left(y_{1}\right)-\sum_{i=1}^{N} S^{\pi(i)}\left(y_{1}\right)\right|^{2} & =\int_{G} \mathbf{E}\left|S\left(g \cdot y_{1}\right)-\sum_{i=1}^{N} S^{\pi(i)}\left(g \cdot y_{1}\right)\right|^{2} d g \\
& =\mathbf{E} \int_{G}\left|S_{G}\left[x_{0}\right](g)-\sum_{i=1}^{N} S_{G}^{\pi(i)}\left[x_{0}\right](g)\right|^{2} d g \underset{N \rightarrow+\infty}{\rightarrow} 0,
\end{aligned}
$$


due to an application of the dominated convergence theorem. Theorem 3 is proved.

When $G$ is finite, formula (28) holds even if the law of $S$ is not $G$ invariant (but, in this case, the series is not necessarily orthogonal in $L^{2}(\mathbf{P})$ ). We now apply Theorem 2 to further characterize actions of the specific form (24). Observe that the following theorem applies to processes whose laws are not necessarily $G$-invariant.

Theorem 4. Let the action $A: G \times X_{0} \mapsto X_{0}$ be such that, for all $(\omega, y) \in X_{0}, A(g,(\omega, y))=\left(\omega, A^{\prime}(g, y)\right)$, where $A^{\prime}$ is an action on $(Y, \mathscr{Y})$. Consider moreover two $Y$-indexed stochastic processes $S, Z$ (not necessarily with $G$-invariant laws), as well as a $\sigma$-finite positive measure $\mu$ on $(Y, \mathscr{Y})$, which is invariant with respect to the action $A^{\prime}$ of $G$ on $Y$. Suppose that, for every fixed $\omega^{*} \in \Omega$, the applications $(g, y) \mapsto Z\left(\omega^{*}, A^{\prime}(g, y)\right)$ and $(g, y) \mapsto$ $S\left(\omega^{*}, A^{\prime}(g, y)\right)$ are elements of $L^{2}(d g \times \mu(d y))$. and also that, for every fixed $\left(\omega^{*}, g^{*}\right) \in \Omega \times G$, the mappings $y \mapsto Z\left(\omega^{*}, A^{\prime}\left(g^{*}, y\right)\right)$ and $y \mapsto S\left(\omega^{*}, A^{\prime}\left(g^{*}, y\right)\right)$ are in $L^{2}(\mu(d y))$. Then,

1) for every fixed $\left(\omega^{*}, g^{*}\right) \in \Omega \times G$, and for every $[\pi],[\sigma] \in \widehat{G}$ such that $[\pi] \neq[\sigma]$

$$
\int_{Y} S_{X_{0}}^{\pi}\left[g^{*}\right]\left(\omega^{*}, y\right) \overline{Z_{X_{0}}^{\sigma}\left[g^{*}\right]\left(\omega^{*}, y\right)} \mu(d y)=0
$$

2) for every fixed $\omega^{*} \in \Omega$,

$$
S\left(\omega^{*}, y\right)=\sum_{[\pi] \in \widehat{G}} S^{\pi}\left(\omega^{*}, y\right) \quad \text { and } \quad Z\left(\omega^{*}, y\right)=\sum_{[\pi] \in \widehat{G}} Z^{\pi}\left(\omega^{*}, y\right)
$$

where the two series are orthogonal and convergent in $L^{2}(\mu(d y))$, and therefore

$$
\left\langle S\left(\omega^{*}, \cdot\right), Z\left(\omega^{*}, \cdot\right)\right\rangle_{L^{2}(\mu(d y))}=\sum_{[\pi] \in \widehat{G}}\left\langle S^{\pi}\left(\omega^{*}, \cdot\right), Z^{\pi}\left(\omega^{*}, \cdot\right)\right\rangle_{L^{2}(\mu(d y))} ;
$$

3) if moreover $Z(\omega, y) \in L^{2}(\mathbf{P}(d \omega) \times \mu(d y))$, then

$$
Z^{\pi}(\omega, y) \in L^{2}(\mathbf{P}(d \omega) \times \mu(d y)), \quad \text { for every }[\pi] \in \widehat{G},
$$

and

$$
Z(\omega, y)=\sum_{[\pi] \in \widehat{G}} Z^{\pi}(\omega, y)
$$

where the series is orthogonal and convergent in $L^{2}(\mathbf{P}(d \omega) \times \mu(d y))$.

P r o o f. 1) For every $\omega^{*} \in \Omega$, the measure $\nu^{*}$ on $\left(X_{0}, \mathscr{X}_{0}\right)=(\Omega \times$ $Y, \mathscr{F} \otimes \mathscr{Y})$, defined by $\nu^{*}(d \omega, d y)=\delta_{\omega^{*}}(d \omega) \times \mu(d y)$, where $\delta_{\omega^{*}}$ is the Dirac mass at $\omega^{*}$, is invariant with respect to the action $A$ of $G$ on $X_{0}$. Moreover, it is easily seen that the assumptions in the statement imply that $\nu^{*}$ satisfies 
all the hypotheses of point 3) of Theorem 2, so that (29) follows immediately, by observing that, for every $g \in G$,

$$
\int_{Y} S_{X_{0}}^{\pi}[g]\left(\omega^{*}, y\right) \overline{Z_{X_{0}}^{\sigma}[g]\left(\omega^{*}, y\right)} \mu(d y)=\int_{X_{0}} S_{X_{0}}^{\pi}[g]\left(x_{0}\right) \overline{Z_{X_{0}}^{\sigma}[g]\left(x_{0}\right)} \nu^{*}\left(d x_{0}\right) .
$$

2) This is a direct consequence of point 5) of Theorem 2 (in the case $g=e)$.

3) First observe that $Z^{\pi}(\omega, y) \in L^{2}(\mathbf{P}(d \omega) \times \mu(d y))$, since, thanks to the $G$-invariance of $\mu$,

$$
\begin{aligned}
\mathbf{E} \int_{Y}\left|Z^{\pi}(y)\right|^{2} \mu(d y) & \leqslant d_{\pi}^{2} \alpha_{\pi}^{2} \int_{Y} \mu(d y) \int_{\Omega} \mathbf{P}(d \omega) \int_{G} d g\left|Z\left(\omega, g^{-1} \cdot y\right)\right|^{2} \\
& =d_{\pi}^{2} \alpha_{\pi}^{2} \int_{G} \int_{Y} \mathbf{E}\left|Z\left(g^{-1} \cdot y\right)\right|^{2} \mu(d y) d g \\
& =d_{\pi}^{2} \alpha_{\pi}^{2} \int_{Y} \mathbf{E}|Z(y)|^{2} \mu(d y)<+\infty .
\end{aligned}
$$

The rest of the proof is similar to the proof of point 4) of Theorem 4. Let indeed $[\pi(i)], i=1,2, \ldots$, be an enumeration of $\widehat{G}$, and observe that point 4 ) of Theorem 2 (formula (18)) implies that, for every $\omega^{*} \in \Omega$,

$$
\begin{aligned}
& \lim _{N \rightarrow+\infty} \int_{Y} \int_{G}\left|Z\left(\omega^{*}, g \cdot y\right)-\sum_{i=1}^{N} Z^{\pi(i)}\left(\omega^{*}, g \cdot y\right)\right|^{2} d g \mu(d y) \\
& \quad=\lim _{N \rightarrow+\infty} \int_{X_{0}} \int_{G}\left|Z\left(g \cdot x_{0}\right)-\sum_{i=1}^{N} Z^{\pi(i)}\left(g \cdot x_{0}\right)\right|^{2} d g \nu^{*}\left(d x_{0}\right)=0
\end{aligned}
$$

and (19) yields also

$$
\begin{aligned}
& \int_{Y} \int_{G}\left|Z\left(\omega^{*}, g \cdot y\right)-\sum_{i=1}^{N} Z^{\pi(i)}\left(\omega^{*}, g \cdot y\right)\right|^{2} d g \mu(d y) \\
& \quad \leqslant \int_{Y} \int_{G}|Z(g \cdot y)|^{2} d g \mu(d y)=\int_{Y}|Z(y)|^{2} \mu(d y) \in L^{1}(\mathbf{P}),
\end{aligned}
$$

since $Z \in L^{2}(\mathbf{P}(d \omega) \times \mu(d y))$ by assumption. Since $\mu$ is $G$-invariant, and by dominated convergence,

$$
\begin{aligned}
\mathbf{E} \int_{Y}\left|Z(y)-\sum_{i=1}^{N} Z^{\pi(i)}(y)\right|^{2} \mu(d y) \\
\quad=\int_{G} \mathbf{E} \int_{Y}\left|Z(g \cdot y)-\sum_{i=1}^{N} Z^{\pi(i)}(g \cdot y)\right|^{2} \mu(d y) d g \\
\quad=\mathbf{E}\left[\int_{Y} \int_{G}\left|Z(g \cdot y)-\sum_{i=1}^{N} Z^{\pi(i)}(g \cdot y)\right|^{2} d g \mu(d y) \underset{N \rightarrow+\infty}{\rightarrow} 0 .\right.
\end{aligned}
$$




\section{Applications to Gaussian processes.}

4.1. Decomposition of real-valued Gaussian processes. Keep the previous notation and assumptions (in particular, Assumption A holds throughout the following). In this section, we apply the above-established results to the case of a two-dimensional real-valued Gaussian process of the type $\left(Z_{1}, Z_{2}\right): \Omega \times Y \rightarrow \mathbf{R}^{2}:(\omega, y) \mapsto\left(Z_{1}(\omega, y), Z_{2}(\omega, y)\right)$ with a covariance structure given by

$$
R_{i, j}\left(y_{1}, y_{2}\right)=\mathbf{E}\left[Z_{i}\left(y_{1}\right) Z_{j}\left(y_{2}\right)\right], \quad i, j=1,2, \quad y_{1}, y_{2} \in Y .
$$

Note that our definition of two-dimensional Gaussian process also covers the case $Z_{1}=Z_{2}$. Here we will consider exclusively actions of the type (24), where $A^{\prime}$ is an action of the topological compact group $G$ on $Y$. Note that, under such assumptions, $\left(Z_{1}, Z_{2}\right)$ has a $G$-invariant law if and only if

$R_{i, j}\left(g \cdot y_{1}, g \cdot y_{2}\right)=R_{i, j}\left(y_{1}, y_{2}\right) \quad$ for every $g \in G, i, j=1,2$, and $y_{1}, y_{2} \in Y$.

When the function $R_{i, j}$ satisfies (34), we say that $R_{i, j}$ is a $G$-invariant covariance function.

In the sequel, the Cartesian product $G \times G=G^{2}$ is systematically endowed with the product group structure, as described, e.g., in [19, Section 3.2]. The generic element of $G^{2}$ is denoted by $\left(g_{1}, g_{2}\right) ; G^{2}$ is again a topological and compact group, with Haar measure given by $d g_{1} \times d g_{2}$. Recall (see again [19, Sections 1.5 and 3.2]) that $[\rho] \in \widehat{G^{2}}$ if and only if $[\rho]=\left[\pi_{1}\right] \otimes\left[\pi_{2}\right]$, where $\left(\left[\pi_{1}\right],\left[\pi_{2}\right]\right) \in \widehat{G} \times \widehat{G}$, and $\otimes$ stands for the (tensor) product between representations. The following assumption will hold for the rest of the section.

A s s u m ption B. For every two-dimensional Gaussian process $\left(Z_{1}, Z_{2}\right)$ considered in the sequel, and for every fixed $y_{1}, y_{2} \in Y$, the application

$$
\left(R_{i, j}\right)_{G^{2}}\left[y_{1}, y_{2}\right]: G \times G \rightarrow \mathbf{R}:\left(g_{1}, g_{2}\right) \mapsto R_{i, j}\left(g_{1} \cdot y_{1}, g_{2} \cdot y_{2}\right)
$$

(see (34), and observe that (35) is consistent with the notation introduced in (7)) is an element of $L^{2}\left(G^{2}\right)$, for every $i, j=1,2$.

Again, if $G$ is finite, Assumption B is redundant. Given $\widehat{G^{2}} \ni[\rho]=$ $\left[\pi_{1}\right] \otimes\left[\pi_{2}\right]$, we define, for fixed $y_{1}, y_{2} \in Y$,

$$
\left(R_{i, j}\right)_{G^{2}}^{\rho}\left[y_{1}, y_{2}\right]=\left(R_{i, j}\right)_{G^{2}}^{\pi_{1} \otimes \pi_{2}}\left[y_{1}, y_{2}\right]
$$

according to (11). The following result, which is a consequence of Theorem 3, will lead to a very general version of Watson's duplication identity.

Proposition 2. Let $\left(Z_{1}, Z_{2}\right)$ be a two-dimensional real-valued Gaussian process with a $G$-invariant law. Then, 
1) the collection of (possibly complex-valued) stochastic processes $\left\{Z_{1}, Z_{2}, Z_{1}^{\pi}, Z_{2}^{\sigma}:[\pi],[\sigma] \in \widehat{G}\right\}$ is jointly Gaussian;

2) for every $[\pi],[\sigma] \in \widehat{G}$ such that $[\pi] \neq[\sigma]$ and $\chi_{\pi}$ is real-valued, the two processes $Z_{i}^{\pi}$ and $Z_{j}^{\sigma}$ are independent for every $i, j=1,2$;

3) for every $[\pi],[\sigma]$ as at point 2$),\left(R_{i, j}\right)_{G^{2}}^{\pi \otimes \sigma}\left[y_{1}, y_{2}\right]=0$, for every $i, j=1,2$;

4) for $i=1,2, Z_{i}(y)=\sum_{[\pi] \in \widehat{G}} Z_{i}^{\pi}(y)$ in $L^{2}(\mathbf{P})$.

P r o o f. Point 1) is immediate, since the action $A$ of $G$ on $X_{0}$ has the form (24). Since $\chi_{\pi}$ is real-valued, $Z_{i}^{\pi}$ is also real-valued, and moreover, for every $y_{1}, y_{2} \in Y$, according to point 3 ) of Theorem $3, \mathbf{E}\left[Z_{i}^{\pi}\left(y_{1}\right) \overline{Z_{j}^{\sigma}\left(y_{2}\right)}\right]=0$, thus implying that $Z_{i}^{\pi}\left(y_{1}\right)$ is independent of both the real and imaginary parts of $Z_{j}^{\sigma}\left(y_{2}\right)$. This concludes the proof of point 2). To see point 3), just write, for $h_{1}, h_{2} \in G$

$$
\begin{aligned}
& \left(R_{i, j}\right)_{G^{2}}^{\pi \otimes \sigma}\left[y_{1}, y_{2}\right]\left(h_{1}, h_{2}\right) \\
& \quad=\int_{G} d g_{1} \int_{G} d g_{2} \chi_{\pi}\left(g_{1}\right) \chi_{\sigma}\left(g_{2}\right)\left(R_{i, j}\right)_{G^{2}}\left[y_{1}, y_{2}\right]\left(g_{1}^{-1} h_{1}, g_{2}^{-1} h_{2}\right) \\
& \quad=\mathbf{E}\left[Z_{i}^{\pi}\left(h_{1} \cdot y_{1}\right) Z_{j}^{\sigma}\left(h_{2} \cdot y_{2}\right)\right]=0,
\end{aligned}
$$

due to point 2). Point 4) comes immediately from point 4) of Theorem 3.

Of course, point 1) of Proposition 1 still holds when the law of the Gaussian process $\left(Z_{1}, Z_{2}\right)$ is not $G$-invariant. The combination of Theorem 4 and Proposition 2 yields immediately the following proposition.

Proposition 3. Let $G$ be such that $\chi_{\pi}$ is real-valued for every $[\pi] \in \widehat{G}$. Let $\left(Z_{1}, Z_{2}\right)$ be a two dimensional real-valued Gaussian process with a $G$ invariant law, and consider a $G$-invariant, $\sigma$-finite and positive measure $\mu$ on $(Y, \mathscr{Y})$. Suppose that, for any fixed $\omega^{*} \in \Omega$ and $i=1,2$, the mapping $(g, y) \mapsto Z_{i}\left(\omega^{*}, g \cdot y\right)$ is in $L^{2}(d g \times \mu(d y))$, and also that, for every fixed $\left(\omega^{*}, g^{*}\right) \in \Omega \times G$, the mapping $y \mapsto Z_{i}\left(\omega^{*}, g^{*} \cdot y\right)$ is an element of $L^{2}(\mu(d y))$. Then, for every $i, j=1,2$,

1) the Gaussian processes $Z_{i}^{\pi}(\omega, y)$ and $Z_{j}^{\sigma}(\omega, y)$ are independent for every $[\pi] \neq[\sigma]$, and orthogonal in $L^{2}(\mu(d y))$ for every $\omega \in \Omega$;

2) for every $[\pi] \in \widehat{G}$,

$$
\mathbf{E}\left[Z_{i}^{\pi}\left(y_{1}\right) Z_{j}^{\pi}\left(y_{2}\right)\right]=\left(R_{i, j}\right)_{G^{2}}^{\pi \otimes \pi}\left[y_{1}, y_{2}\right](e, e)=R_{i, j}^{\pi \otimes \pi}\left(y_{1}, y_{2}\right) ;
$$

3) $Z_{i}(\omega, y)=\sum_{[\pi] \in \widehat{G}} Z_{i}^{\pi}(\omega, y)$ both in $L^{2}(\mu(d y))$ for every $\omega \in \Omega$ and in $L^{2}(\mathbf{P}(d \omega) \times \mu(d y))$

4) for every $\lambda \in \mathbf{R}$,

$$
\begin{aligned}
\mathbf{E}\left[\exp \left(i \lambda \int_{Y} Z_{i}(y) Z_{j}(y) \mu(d y)\right)\right] \\
\quad=\prod_{[\pi] \in \widehat{G}} \mathbf{E}\left[\exp \left(i \lambda \int_{Y} Z_{i}^{\pi}(y) Z_{j}^{\pi}(y) \mu(d y)\right)\right] .
\end{aligned}
$$


E $x$ a m p l e (a group-theoretic proof of the [polarized] Watson's identity). As a first illustration of our techniques, we shall obtain a class of identities in law - between functionals of two correlated Brownian bridges extending Watson's identity (1). Our method of proof, which is directly based on the discussion contained in this section, generalizes the simple proof of (1) given by the second author in [14], and will motivate the content of the subsequent section. To this end, we consider a two-dimensional Brownian bridge $\underline{b}=\left\{b_{1}(t), b_{2}(t): t \in[0,1]\right\}$ with correlation parameter equal to $\rho \in[0,1]$. This means that $\underline{b}$ is a two-dimensional, real-valued Gaussian process such that, for every $s, t \in[0,1], \mathbf{E}\left[b_{i}(s) b_{i}(t)\right]=s \wedge t-s t, i=1,2$, and $\mathbf{E}\left[b_{1}(s) b_{2}(t)\right]=\rho(s \wedge t-s t)$. By $\underline{b}_{*}=\left\{b_{* 1}(t), b_{* 2}(t): t \in[0,1]\right\}$, we denote an independent copy of $\underline{b}$, and we also write, for $i=1,2$ and $t \in[0,1]$,

$$
v_{i}(t)=b_{i}(t)-\int_{0}^{1} b_{i}(s) d s \quad \text { and } \quad v_{* i}(t)=b_{* i}(t)-\int_{0}^{1} b_{* i}(s) d s .
$$

Now consider the group $G=\{e, g\} \simeq \mathbf{Z} / 2 \mathbf{Z}$, where $e$ stands again for the identity element. It is plain (see, e.g., [19, Chap. 2]) that in this case $\widehat{G}=\left\{\left[\pi_{u}\right],\left[\pi_{a}\right]\right\}$, where $\left[\pi_{u}\right]$ and $\left[\pi_{a}\right]$ are the equivalence classes, respectively, of the unity and of the alternating representation; in particular, $\chi_{\pi_{u}}(e)=\chi_{\pi_{u}}(g)=1$, and $\chi_{\pi_{a}}(e)=1=-\chi_{\pi_{a}}(g)$. We fix the following elementary action of $G$ on $[0,1]: e \cdot t=t$ and $g \cdot t=1-t$, for all $t \in[0,1]$. It is well known that $\underline{b}$, and therefore the vector $\left(v_{1}, v_{2}\right)$, has a $G$-invariant law, so that the content of Proposition 2 can be applied directly. To do this, we first set, according to (12) and for $i=1,2$ and $t \in[0,1]$, and since $d_{\pi_{u}}=d_{\pi_{a}}=1$,

$$
\begin{aligned}
v_{i}^{\pi_{u}}(t) & =\frac{d_{\pi_{u}}}{|G|}\left\{\chi_{\pi_{u}}(e) v_{i}\left(e^{-1} \cdot t\right)+\chi_{\pi_{u}}(g) v_{i}\left(g^{-1} \cdot x\right)\right\} \\
& =\frac{1}{2}\left(b_{i}(t)+b_{i}(1-t)\right)-\int_{0}^{1} b_{i}(s) d s \\
& =\frac{1}{2}\left(b_{i}(t)+b_{i}(1-t)\right)-\frac{1}{2} \int_{0}^{1}\left(b_{i}(s)+b_{i}(1-s)\right) d s \\
v_{i}^{\pi_{a}}(t) & =\frac{d_{\pi_{a}}}{|G|}\left\{\chi_{\pi_{a}}(e) v_{i}\left(e^{-1} \cdot t\right)+\chi_{\pi_{a}}(g) v_{i}\left(g^{-1} \cdot x\right)\right\}=\frac{1}{2}\left(b_{i}(t)-b_{i}(1-t)\right),
\end{aligned}
$$

and an analogous definition holds for $v_{* i}^{\pi_{u}}$ and $v_{* i}^{\pi_{a}}, i=1,2$. Now observe that Proposition 2 (in the case $\left.\left(Z_{1}, Z_{2}\right)=\left(v_{1}, v_{2}\right)\right)$ implies that, for any $i, j=1,2$, the two processes $v_{i}^{\pi_{u}}$ and $v_{j}^{\pi_{a}}$ are independent. Moreover, the restriction of Lebesgue measure to $[0,1]$ is trivially $G$-invariant, so that all assumptions of Proposition 3 are satisfied (again with $\left(Z_{1}, Z_{2}\right)=\left(v_{1}, v_{2}\right)$ and $\mu$ equal to Lebesgue measure) and therefore

$$
\int_{0}^{1} v_{1}(t) v_{2}(t) d t \stackrel{\text { law }}{=} \int_{0}^{1} v_{1}^{\pi_{u}}(t) v_{2}^{\pi_{u}}(t) d t+\int_{0}^{1} v_{* 1}^{\pi_{a}}(t) v_{* 2}^{\pi_{a}}(t) d t
$$




$$
\begin{aligned}
=\frac{1}{4} \int_{0}^{1} & \left(b_{1}(t)+b_{1}(1-t)-\int_{0}^{1}\left(b_{1}(s)+b_{1}(1-s)\right) d s\right) \\
& \times\left(b_{2}(t)+b_{2}(1-t)-\int_{0}^{1}\left(b_{2}(s)+b_{2}(1-s)\right) d s\right) d t \\
& +\frac{1}{4} \int_{0}^{1}\left(b_{* 1}(t)-b_{* 1}(1-t)\right)\left(b_{* 2}(t)-b_{* 2}(1-t)\right) d t .
\end{aligned}
$$

Next, consider a correlated two-dimensional standard Brownian motion $\underline{W}=$ $\left\{W_{1}(t), W_{2}(t): t \in[0,1]\right\}$ with correlation $\rho,{ }^{2)}$ and independent of $\underline{b}$. Routine computations show the following identities in law:

$$
\begin{gathered}
\left\{b_{1}(t)+b_{1}(1-t), b_{2}(t)+b_{2}(1-t): t \in[0,1 / 2]\right\} \\
\stackrel{\text { law }}{=}\left\{W_{1}(2 t), W_{2}(2 t): t \in[0,1 / 2]\right\}, \\
\left\{b_{1}(t)-b_{1}(1-t), b_{2}(t)-b_{2}(1-t): t \in[0,1 / 2]\right\} \\
\stackrel{\text { law }}{=}\left\{b_{1}(2 t), b_{2}(2 t): t \in[0,1 / 2]\right\},
\end{gathered}
$$

implying that

$$
\begin{aligned}
\int_{0}^{1} v_{1}(t) v_{2}(t) \stackrel{\text { law }}{=} & \frac{1}{4} \int_{0}^{1}\left(W_{1}(t)-\int_{0}^{1} W_{1}(s) d s\right)\left(W_{2}(t)-\int_{0}^{1} W_{2}(s) d s\right) d t \\
& +\frac{1}{4} \int_{0}^{1} b_{1}(t) b_{2}(t) d t .
\end{aligned}
$$

We eventually use some standard arguments (see, e.g., [2, Lemma 2]) to prove that

$$
\int_{0}^{1}\left(W_{1}(t)-\int_{0}^{1} W_{1}(s) d s\right)\left(W_{2}(t)-\int_{0}^{1} W_{2}(s) d s\right) d t \stackrel{\text { law }}{=} \int_{0}^{1} b_{1}(t) b_{2}(t) d t
$$

and therefore

$$
\begin{gathered}
\int_{0}^{1}\left(b_{1}(t)-\int_{0}^{1} b_{1}(s) d s\right)\left(b_{2}(t)-\int_{0}^{1} b_{2}(s) d s\right) d t \\
\stackrel{\text { law }}{=} \frac{1}{4} \int_{0}^{1}\left(b_{1}(t) b_{2}(t)+b_{* 1}(t) b_{* 2}(t)\right) d t
\end{gathered}
$$

(Watson's identity (1) can be obtained by setting $\rho=1$ ).

R e m a r k. By using, e.g., [2, Proposition 2], we obtain that, for $\lambda>0$ sufficiently small and $\rho \in[0,1]$

$$
\begin{gathered}
\mathbf{E}\left[\exp \left(\lambda^{2} \int_{0}^{1}\left(b_{1}(t)-\int_{0}^{1} b_{1}(s) d s\right)\left(b_{2}(t)-\int_{0}^{1} b_{2}(s) d s\right) d t\right)\right] \\
=\frac{(\lambda / 2)^{2} \sqrt{1-\rho^{2}}}{\sin (\lambda \sqrt{1+\rho} / 2) \operatorname{sh}(\lambda \sqrt{1-\rho} / 2)} .
\end{gathered}
$$

2) That is, $\underline{W}$ is a two-dimensional Gaussian process such that, for $i=1,2$ and $s, t \in[0,1], \mathbf{E}\left[W_{i} \overline{(s)} W_{i}(t)\right]=s \wedge t$ and $\mathbf{E}\left[W_{1}(s) W_{2}(t)\right]=\rho(s \wedge t)$. 
Note that the $G$-invariant process $\left(v_{1}, v_{2}\right)$, introduced in (39) of the previous example, has the remarkable property that

$$
\int_{0}^{1} v_{1}^{\pi_{u}}(t) v_{2}^{\pi_{u}}(t) d t \stackrel{\text { law }}{=} \int_{0}^{1} v_{* 1}^{\pi_{a}}(t) v_{* 2}^{\pi_{a}}(t) d t .
$$

In the next subsection we shall establish necessary and sufficient conditions to ensure that, in the case of a finite $G$, a $G$-invariant Gaussian process $\left(Z_{1}, Z_{2}\right)$ (with some special structure) is such that

$$
\int_{Y} Z_{1}^{\pi}(y) Z_{2}^{\pi}(y) \mu(d y) \stackrel{\text { law }}{=} \int_{Y} Z_{1}^{\sigma}(y) Z_{2}^{\sigma}(y) \mu(d y), \quad \text { for every }[\pi],[\sigma] \in \widehat{G} .
$$

In the sequel, an identity like (41) will be called a Watson's type relation.

4.2. Watson's type relations for Volterra processes. Throughout this subsection, $G$ stands for a finite group such that the character $\chi_{\pi}(\cdot)$ is real-valued for every $[\pi] \in \widehat{G}$. To simplify some technical points of our discussion (in particular, to apply several crucial properties of multiple Wiener-Itô integrals) we will consider a two-dimensional real-valued Gaussian process $\left(Z_{1}, Z_{2}\right)$ such that its components are correlated Volterra processes. To define such objects, take a measurable space $(T, \mathscr{T}, \tau)$, where $\tau$ is positive, $\sigma$-finite and nonatomic, and write $L_{\mathbf{R}}^{2}(d \tau)$ to indicate the Hilbert space of real-valued, square-integrable functions with respect to $\tau$. In what follows, we will write

$$
X=\left\{X(f): f \in L_{\mathbf{R}}^{2}(d \tau)\right\}
$$

to indicate an isonormal Gaussian process (or a Gaussian measure) on $L_{\mathbf{R}}^{2}(d \tau)$. This means that $X$ is a centered Gaussian family indexed by the elements of $L_{\mathbf{R}}^{2}(d \tau)$, defined on some probability space $(\Omega, \mathscr{F}, \mathbf{P})$ and such that, for every $f_{1}, f_{2} \in L_{\mathbf{R}}^{2}(d \tau)$,

$$
\mathbf{E}\left(X\left(f_{1}\right) X\left(f_{2}\right)\right)=\int_{T} f_{1}(t) f_{2}(t) \tau(d t) .
$$

Now fix a measurable space $(Y, \mathscr{Y})$. A two-dimensional Gaussian process $\left\{\left(Z_{1}(y), Z_{2}(y)\right): y \in Y\right\}$ is called a correlated (generalized) Volterra process, with respect to $X$ and with parameter $\rho \in[0,1]$, if there exist two $(\mathscr{Y} \otimes \mathscr{T})$-measurable applications

$$
Y \times T \rightarrow \mathbf{R}:(y, t) \mapsto \phi_{i}(y, t), \quad i=1,2,
$$

such that: (a) for every $y \in Y$ the application $t \mapsto \phi_{i}(y, t)$ is an element of $L_{\mathbf{R}}^{2}(d \tau)$,

$$
\text { (b) } \quad Z_{i}(y)=X\left(\phi_{i}(y, \cdot)\right) \quad \mathbf{P} \text {-a.s., } \quad i=1,2,
$$

and (c) for every $y_{1}, y_{2} \in Y$ and by using the notation introduced in (33),

$$
\begin{aligned}
& R_{1,1}\left(y_{1}, y_{2}\right)=R_{2,2}\left(y_{1}, y_{2}\right) \quad \text { and } \\
& R_{1,2}\left(y_{1}, y_{2}\right)=R_{2,1}\left(y_{1}, y_{2}\right)=\rho R_{1,1}\left(y_{1}, y_{2}\right) .
\end{aligned}
$$


Note that, if $\rho=1$, then $Z_{1}(y)=Z_{2}(y) \mathbf{P}$-a.s., for all $y \in Y$; moreover, the covariance structure of a Gaussian process $\left(Z_{1}, Z_{2}\right)$ of the type (43) may be rewritten as

$$
R_{i, j}\left(y_{1}, y_{2}\right)=\mathbf{E}\left[Z_{i}\left(y_{1}\right) Z_{j}\left(y_{2}\right)\right]=\int_{T} \phi_{i}\left(y_{1}, t\right) \phi_{j}\left(y_{2}, t\right) \tau(d t), \quad i, j=1,2 ;
$$

as a consequence, in view of (44) and (45), and given an action $g \cdot y$ of $G$ on $Y$, the process $\left(Z_{1}, Z_{2}\right)$ has a $G$-invariant law if and only if, for $i$ equal to 1 or 2 ,

$$
\int_{T} \phi_{i}\left(g \cdot y_{1}, t\right) \phi_{i}\left(g \cdot y_{2}, t\right) \tau(d t)=\int_{T} \phi_{i}\left(y_{1}, t\right) \phi_{i}\left(y_{2}, t\right) \tau(d t),
$$

for every $y_{1}, y_{2} \in Y$ and every $g \in G$. In the sequel, to simplify the notation, we will write

$$
R_{1,1}(\cdot, \cdot)=R_{2,2}(\cdot, \cdot)=R(\cdot, \cdot) .
$$

We now fix an action $g \cdot y$ of $G$ on $Y$, as well as a $G$-invariant, positive, and $\sigma$-finite measure $\mu$ on $(Y, \mathscr{Y})$. For every real-valued $\Phi, \Psi \in$ $L^{2}\left(Y^{2}, \mathscr{Y}^{2}, d \mu \times d \mu\right):=L^{2}(d \mu \times d \mu)$, we define, for $y_{1}, y_{2} \in Y$,

(i) $\left[\Phi \otimes_{(1)} \Phi\right]\left(y_{1}, y_{2}\right)=\Phi\left(y_{1}, y_{2}\right)$;

(ii) $\left[\Phi \otimes_{(2)} \Psi\right]\left(y_{1}, y_{2}\right)=\int_{Y} \Phi\left(y_{1}, x\right) \Psi\left(y_{2}, x\right) \mu(d x)$;

(iii) for all $p \geqslant 3,\left[\Phi \otimes_{(p)} \Phi\right]\left(y_{1}, y_{2}\right)=\left[\left[\Phi \otimes_{(p-1)} \Phi\right] \otimes_{(2)} \Phi\right]\left(y_{1}, y_{2}\right)$.

Observe that, if $\Phi \in L^{2}(d \mu \times d \mu)$, then $y \mapsto\left[\Phi \otimes_{(p)} \Phi\right](y, y)$ is an element of $L^{1}(Y, \mathscr{Y}, d \mu)$ for every $p \geqslant 2$. Finally, for $\rho \in[0,1]$ as above, we introduce the following set of real constants:

$$
\begin{aligned}
& K(1, \rho)=2 \rho \\
& K(n, \rho)=2 \sum_{j=0}^{n / 2-1}\left(\begin{array}{c}
n-1 \\
2 j
\end{array}\right) \rho^{2 j}+2 \sum_{j=0}^{n / 2-1}\left(\begin{array}{c}
n-1 \\
2 j+1
\end{array}\right) \rho^{2 j+2}, \quad n \geqslant 2 \text { even, }(48) \\
& K(n, \rho)=2 \sum_{j=0}^{(n-1) / 2}\left(\begin{array}{c}
n-1 \\
2 j
\end{array}\right) \rho^{2 j+1}+2 \sum_{j=0}^{(n-3) / 2}\left(\begin{array}{c}
n-1 \\
2 j+1
\end{array}\right) \rho^{2 j+1}, \quad n \geqslant 3 \text { odd. }
\end{aligned}
$$

Note that $K(n, 1)=2^{n}$ for every $n \geqslant 1, K(2 p, \rho)>0$ for every $p \geqslant 1$, and, for $p \geqslant 0, K(2 p+1, \rho)=0$ if and only if $\rho=0$ (since $\rho$ is real). In the next result, under some additional integrability assumptions, we state necessary and sufficient conditions for property (41) to be satisfied.

Theorem 5. Consider a finite group $G$ such that $\chi_{\pi}(\cdot) \in \mathbf{R}$, for every $[\pi] \in \widehat{G}$. Let the process $\left(Z_{1}, Z_{2}\right)$ be a correlated Volterra process of the type (43), for some correlation coefficient $\rho \in[0,1]$, and assume $\left(Z_{1}, Z_{2}\right)$ has a $G$-invariant law. Let also $\mu(\cdot)$ be a $G$-invariant positive measure satisfying the assumptions of Proposition 3, and suppose moreover

$\mathbf{E}\left(\int_{Y} Z_{1}(y)^{2} \mu(d y)\right)=\int_{Y} \int_{T} \phi_{1}(y, t)^{2} \mu(d y) \tau(d t)=\int_{Y} R(y, y) \mu(d y)<+\infty$. 
Then,

1 ) the covariance functions $R$ and $R^{\pi \otimes \pi}$ defined, respectively, according to (47) and (37), for $[\pi] \in \widehat{G}$, satisfy

$$
\int_{Y} \int_{Y} R(x, y)^{2} \mu(d x) \mu(d y)<+\infty, \quad \int_{Y} \int_{Y} R^{\pi \otimes \pi}(x, y)^{2} \mu(d x) \mu(d y)<+\infty ;
$$

2) the random variables $\int_{Y} Z_{1}^{\pi}(y) Z_{2}^{\pi}(y) \mu(d y),[\pi] \in \widehat{G}$, are stochastically independent;

3) for every $[\pi] \in \widehat{G}$, the process $\left(Z_{1}^{\pi}, Z_{2}^{\pi}\right)$ is a correlated Volterra process with parameter $\rho$;

4) the following three conditions are equivalent: (i) for every $[\pi],[\sigma] \in \widehat{G}$,

$$
\int_{Y} Z_{1}^{\pi}(y) Z_{2}^{\pi}(y) \mu(d y) \stackrel{\text { law }}{=} \int_{Y} Z_{1}^{\sigma}(y) Z_{2}^{\sigma}(y) \mu(d y),
$$

(ii) for every $[\pi] \in \widehat{G}$ and every $n \geqslant 1$

$$
K(n, \rho) \int_{Y}\left[R^{\pi \otimes \pi} \otimes_{(n)} R^{\pi \otimes \pi}\right](y, y) \mu(d y)=\frac{K(n, \rho)}{|\widehat{G}|} \int_{Y}\left[R \otimes_{(n)} R\right](y, y) \mu(d y),
$$

(iii) for every $[\pi] \in \widehat{G}$ and every $n \geqslant 1$

$$
\begin{aligned}
& K(n, \rho) \int_{Y}\left[R^{\pi \otimes \pi} \otimes_{(n)} R^{\pi \otimes \pi}\right](y, y) \mu(d y) \\
& \quad=K(n, \rho) \int_{Y}\left[R^{\sigma \otimes \sigma} \otimes_{(n)} R^{\sigma \otimes \sigma}\right](y, y) \mu(d y) .
\end{aligned}
$$

$\mathrm{R}$ e m a r k. (i) In view of (45), both formulae (52) and (53) can immediately be reformulated in terms of the kernels $\phi_{1}$ and $\phi_{2}$.

(ii) The role of the constants $K(n, \rho)$ in (52) and (53) is immaterial for $\rho \neq 0$, or for $n$ even and $\rho \in[0,1]$.

Before proving Theorem 5, we state some interesting consequences of point 4) of Theorem 5 .

Proposition 4. Let $G=\{e, g\} \simeq \mathbf{Z} / 2 \mathbf{Z}$, where e stands for the identity element. Keep the assumptions and the notation of Theorem 5 , and suppose moreover that $\rho \neq 0$. Then, condition (51) is verified if and only if for every $n \geqslant 1$

$$
\int_{Y}\left[R \otimes_{(n)} R\right](y, g \cdot y) \mu(d y)=0 .
$$

P r o o f. As already pointed out, in this case $\widehat{G}=\left\{\left[\pi_{u}\right],\left[\pi_{a}\right]\right\}$, where $\left[\pi_{u}\right]$ and $\left[\pi_{a}\right]$ are the equivalence classes of the unity and of the alternating representation. Moreover, due to Theorem 5 and the fact that $K(n, \rho) \neq 0$, we know that (51) holds if and only if

$$
\int_{Y}\left[R^{\pi_{u} \otimes \pi_{u}} \otimes_{(n)} R^{\pi_{u} \otimes \pi_{u}}\right](y, y) \mu(d y)=\frac{1}{2} \int_{Y}\left[R \otimes_{(n)} R\right](y, y) \mu(d y),
$$


for any $n \geqslant 1$, where

$$
\begin{aligned}
R^{\pi_{u} \otimes \pi_{u}}\left(y_{1}, y_{2}\right)= & \frac{1}{4}\left(R\left(e \cdot y_{1}, e \cdot y_{2}\right)+R\left(g \cdot y_{1}, e \cdot y_{2}\right)\right. \\
& \left.+R\left(e \cdot y_{1}, g \cdot y_{2}\right)+R\left(g \cdot y_{1}, g \cdot y_{2}\right)\right) \\
= & \frac{1}{2}\left(R\left(y_{1}, y_{2}\right)+R\left(y_{1}, g \cdot y_{2}\right)\right),
\end{aligned}
$$

due to the $G$-invariance of the law of $\left(Z_{1}, Z_{2}\right)$. Finally, since $\mu$ is also $G$ invariant, one can easily prove that, for $n \geqslant 1$,

$$
\begin{aligned}
\int_{Y}\left[R^{\pi_{u} \otimes \pi_{u}} \otimes_{(n)} R^{\pi_{u} \otimes \pi_{u}}\right](y, y) \mu(d y)= & \frac{1}{2} \int_{Y}\left[R \otimes_{(n)} R\right](y, y) \mu(d y) \\
& +\frac{1}{2} \int_{Y}\left[R \otimes_{(n)} R\right](y, g \cdot y) \mu(d y),
\end{aligned}
$$

thus yielding, via (54), the desired conclusion.

$\mathrm{R} \mathrm{e} \mathrm{m}$ a $\mathrm{rk}$. The process $\left(v_{1}, v_{2}\right)$ defined in (39) can be represented as a correlated Volterra process with parameter $\rho \in[0,1]$ and covariance structure

$$
\begin{aligned}
\mathbf{E}\left[v_{1}(s) v_{1}(t)\right] & =\mathbf{E}\left[v_{2}(s) v_{2}(t)\right]=R_{v}(s, t) \\
& =s \wedge t-\frac{s+t}{2}+\frac{(s-t)^{2}}{2}+\frac{1}{12}, \\
\mathbf{E}\left[v_{1}(s) v_{2}(t)\right] & =\mathbf{E}\left[v_{2}(s) v_{1}(t)\right]=\rho R_{v}(s, t),
\end{aligned}
$$

where $s, t \in[0,1]$. Moreover, its law is $G$-invariant, where $G=\mathbf{Z} / 2 \mathbf{Z}$. Since (40) holds, we deduce from Proposition 4 that for every $n \geqslant 1$

$$
\int_{0}^{1}\left[R_{v} \otimes_{(n)} R_{v}\right](t, 1-t) d t=0
$$

The following result, which is again a consequence of Theorem 5 , is very useful to deal with multiparameter processes.

Proposition 5. Fix $d \geqslant 2$. Let $\left(Y^{(i)}, \mathscr{Y}^{(i)}, \mu^{(i)}\right), i=1, \ldots, d$, be a collection of measure spaces, with $\mu^{(i)}$ positive and $\sigma$-finite, and let $G^{(i)}, \ldots, G^{(d)}$ be finite groups with real-valued characters, such that, for each $i=1, \ldots, d$, an action $g_{i} \cdot y_{i}$ of $G_{i}$ on $Y_{i}$ is well defined. We use the notation

$$
\begin{aligned}
Y & =Y^{(1)} \times \cdots \times Y^{(d)}, \quad G=G^{(1)} \times \cdots \times G^{(d)}, \\
\mu & =\mu^{(1)} \times \cdots \times \mu^{(d)}
\end{aligned}
$$

and we endow $G$ with the product group structure (see [19, Section 3.2]). Let also $\left\{\left(Z_{1}(y), Z_{2}(y)\right): y \in Y\right\}$ be a correlated Volterra process with parameter $\rho \in[0,1]$, such that, for every $y=\left(y_{1}, \ldots, y_{d}\right)$ and $x=\left(x_{1}, \ldots, x_{d}\right)$ in $Y$,

$\mathbf{E}\left[Z_{1}(x) Z_{1}(y)\right]=R(x, y)=\prod_{i=1}^{d} R_{i}\left(x_{i}, y_{i}\right) \quad$ and $\quad \mathbf{E}\left[Z_{1}(x) Z_{2}(y)\right]=\rho R(x, y)$, 
where for each $i, R_{i}$ is a $G^{(i)}$-invariant covariance function such that $\int_{Y^{(i)}} R_{i}(y, y) \mu^{(i)}(d y)<+\infty$. Then,

1) the application $(g, y) \mapsto g \cdot y:\left(g_{1}, \ldots, g_{d} ; y_{1}, \ldots, y_{d}\right) \mapsto\left(g_{1} \cdot y_{1}, \ldots\right.$, $\left.g_{d} \cdot y_{d}\right)$ is an action of $G$ on $Y$;

2) the process $\left(Z_{1}, Z_{2}\right)$ has a $G$-invariant law;

3) for every $\left[\pi_{i}\right],\left[\sigma_{i}\right] \in \widehat{G^{(i)}}, i=1, \ldots, d$, and every $x, y \in Y$

$$
R^{\theta}(x, y)=\left\{\begin{array}{l}
0 \quad \text { if there exists } i \text { such that }\left[\pi_{i}\right] \neq\left[\sigma_{i}\right] \\
\prod_{i=1}^{d} R^{\pi_{i} \otimes \pi_{i}}\left(x_{i}, y_{i}\right) \quad \text { otherwise }
\end{array}\right.
$$

where $\theta=\left[\pi_{i} \otimes \cdots \otimes \pi_{d}\right] \otimes\left[\sigma_{i} \otimes \cdots \otimes \sigma_{d}\right]$ is a generic element of $\widehat{G^{2}}$;

4) if, for each $i=1, \ldots, d$, the function $R_{i}$ satisfies either (52) or (53), then $\left(Z_{1}, Z_{2}\right)$ verifies Watson's relation (51) for every $[\pi],[\sigma] \in \widehat{G}$.

P r o o f. Points 1) and 2) are trivial. Point 3) is a consequence of the $G^{(i)}$-invariance of each $R_{i}$, as well as of point 3) of Proposition 2. To prove point 4), suppose that each $R_{i}$ verifies (53), and also $\rho \neq 0$. Then, $K(n, \rho) \neq 0$ for each $n$, and

$$
\begin{gathered}
\frac{1}{|\widehat{G}|} \int_{Y}\left[R \otimes_{(n)} R\right](y, y) \mu(d y)=\frac{1}{|\widehat{G}|} \prod_{i=1}^{d} \int_{Y^{(i)}}\left[R_{i} \otimes_{(n)} R_{i}\right]\left(y_{i}, y_{i}\right) \mu^{(i)}\left(d y_{i}\right) \\
=\prod_{i=1}^{d} \int_{Y^{(i)}}\left[R_{i}^{\left[\pi_{i}\right] \otimes\left[\pi_{i}\right]} \otimes_{(n)} R_{i}^{\left[\pi_{i}\right] \otimes\left[\pi_{i}\right]}\right]\left(y_{i}, y_{i}\right) \mu^{(i)}\left(d y_{i}\right),
\end{gathered}
$$

for every $\left[\pi_{i}\right] \in \widehat{G^{(i)}}, i=1, \ldots, d$, since $|\widehat{G}|=\prod_{i=1, \ldots, d}\left|\widehat{G^{(i)}}\right|$. To conclude, just observe that, thanks to point 3),

$$
\begin{gathered}
\prod_{i=1}^{d} \int_{Y^{(i)}}\left[R_{i}^{\left[\pi_{i}\right] \otimes\left[\pi_{i}\right]} \otimes_{(n)} R_{i}^{\left[\pi_{i}\right] \otimes\left[\pi_{i}\right]}\right]\left(y_{i}, y_{i}\right) \mu^{(i)}\left(d y_{i}\right) \\
=\int_{Y}\left[R^{\eta \otimes \eta} \otimes_{(n)} R^{\eta \otimes \eta}\right](y, y) \mu(d y),
\end{gathered}
$$

where $\eta=\pi_{i} \otimes \cdots \otimes \pi_{d}$ (to deal with the case $\rho=0$, just perform the same argument for even cumulants).

Ex a m p l e (a quadruplication identity). Let $\mathbb{B}_{0}=\left\{\mathbb{B}_{0,1}\left(t_{1}, t_{2}\right)\right.$, $\left.\mathbb{B}_{0,2}\left(t_{1}, t_{2}\right):\left(t_{1}, t_{2}\right) \in[0,1]^{2}\right\}$ be a correlated tied-down Brownian sheet, that is, $\mathbb{B}_{0}$ is a two-dimensional Gaussian process such that

$$
\begin{aligned}
\mathbf{E}\left[\mathbb{B}_{0,1}\left(t_{1}, t_{2}\right), \mathbb{B}_{0,1}\left(s_{1}, s_{2}\right)\right] & =\mathbf{E}\left[\mathbb{B}_{0,2}\left(t_{1}, t_{2}\right), \mathbb{B}_{0,2}\left(s_{1}, s_{2}\right)\right] \\
& =\left(t_{1} \wedge s_{1}-s_{1} t_{1}\right)\left(t_{2} \wedge s_{2}-s_{2} t_{2}\right), \\
\mathbf{E}\left[\mathbb{B}_{0,1}\left(t_{1}, t_{2}\right), \mathbb{B}_{0,2}\left(s_{1}, s_{2}\right)\right] & =\mathbf{E}\left[\mathbb{B}_{0,2}\left(t_{1}, t_{2}\right), \mathbb{B}_{0,1}\left(s_{1}, s_{2}\right)\right] \\
& =\rho\left(t_{1} \wedge s_{1}-s_{1} t_{1}\right)\left(t_{2} \wedge s_{2}-s_{2} t_{2}\right),
\end{aligned}
$$


where $\left(s_{1}, s_{2}\right),\left(t_{1}, t_{2}\right) \in[0,1]^{2}$ and $\rho \in[0,1]$. Note that $\mathbb{B}_{0}$ can be represented as a Volterra process. Moreover, $\mathbb{B}_{0}$ has the law of a correlated Brownian sheet $\mathbb{W}$ (with the same parameter ${ }^{3)}$ ), conditioned to vanish on the edges of the square $[0,1]^{2}$. Now define, for $i=1,2$, the compensated processes

$$
\begin{aligned}
\mathbb{U}_{i}\left(t_{1}, t_{2}\right)= & \mathbb{B}_{0, i}\left(t_{1}, t_{2}\right)-\int_{0}^{1} \mathbb{B}_{0, i}\left(t_{1}, u_{2}\right) d u_{2}-\int_{0}^{1} \mathbb{B}_{0, i}\left(u_{1}, t_{2}\right) d u_{1} \\
& +\int_{[0,1]^{2}} \mathbb{B}_{0, i}\left(u_{1}, u_{2}\right) d u_{1} d u_{2},
\end{aligned}
$$

where $\left(t_{1}, t_{2}\right) \in[0,1]^{2}$. We claim that the following identity in law holds:

$$
\begin{aligned}
\int_{[0,1]^{2}} & \mathbb{U}_{1}\left(t_{1}, t_{2}\right) \mathbb{U}_{2}\left(t_{1}, t_{2}\right) d t_{1} d t_{2} \\
& \stackrel{\text { law }}{=} \frac{1}{16} \sum_{i=1}^{4} \int_{[0,1]^{2}} \mathbb{B}_{0,1}^{(i)}\left(t_{1}, t_{2}\right) \mathbb{B}_{0,2}^{(i)}\left(t_{1}, t_{2}\right)\left(t_{1}, t_{2}\right)^{2} d t_{1} d t_{2},
\end{aligned}
$$

where $\mathbb{B}_{0}^{(i)}=\left(\mathbb{B}_{0,1}^{(i)}, \mathbb{B}_{0,2}^{(i)}\right), i=1, \ldots, 4$, are four independent copies of $\mathbb{B}_{0}$. As a matter of fact, standard calculations show that

$$
\begin{aligned}
& \mathbf{E}\left[\mathbb{U}_{1}\left(t_{1}, t_{2}\right) \mathbb{U}_{1}\left(s_{1}, s_{2}\right)\right]=\mathbf{E}\left[\mathbb{U}_{2}\left(t_{1}, t_{2}\right) \mathbb{U}_{2}\left(s_{1}, s_{2}\right)\right]=R_{v}\left(s_{1}, t_{1}\right) R_{v}\left(s_{2}, t_{2}\right), \\
& \mathbf{E}\left[\mathbb{U}_{1}\left(t_{1}, t_{2}\right) \mathbb{U}_{2}\left(s_{1}, s_{2}\right)\right]=\mathbf{E}\left[\mathbb{U}_{2}\left(t_{1}, t_{2}\right) \mathbb{U}_{1}\left(s_{1}, s_{2}\right)\right]=\rho R_{v}\left(s_{1}, t_{1}\right) R_{v}\left(s_{2}, t_{2}\right),
\end{aligned}
$$

where $R_{v}$ is defined as in (55). Since $R_{v}$ is invariant with respect to the action of $\{e, g\} \simeq(\mathbf{Z} / 2 \mathbf{Z})$ on $[0,1]$ given by $e \cdot t=t$ and $g \cdot t=1-t$, point 2$)$ of Proposition 5 entails that the law of the vector $\left(\mathbb{U}_{1}, \mathbb{U}_{2}\right)$ is invariant with respect to the action of the product group $G=\{e, g\} \times\{e, g\} \simeq(\mathbf{Z} / 2 \mathbf{Z}) \times$ $(\mathbf{Z} / 2 \mathbf{Z})$ on $[0,1]^{2}$ defined as

$$
\begin{aligned}
(e, e) \cdot\left(t_{1}, t_{2}\right) & =\left(t_{1}, t_{2}\right), & (e, g) \cdot\left(t_{1}, t_{2}\right) & =\left(t_{1}, 1-t_{2}\right), \\
(g, e) \cdot\left(1-t_{1}, t_{2}\right) & =\left(t_{1}, t_{2}\right), & (g, g) \cdot\left(t_{1}, t_{2}\right) & =\left(1-t_{1}, 1-t_{2}\right) .
\end{aligned}
$$

Now recall that $\widehat{G}=\left\{\left[\pi_{u}\right] \otimes\left[\pi_{u}\right],\left[\pi_{a}\right] \otimes\left[\pi_{u}\right],\left[\pi_{u}\right] \otimes\left[\pi_{a}\right],\left[\pi_{a}\right] \otimes\left[\pi_{a}\right]\right\}$, where $\pi_{u}$ and $\pi_{a}$ are the unity and alternating representations of $\mathbf{Z} / 2 \mathbf{Z}$. According to point 4 ) of Proposition 3 (since Lebesgue measure on $[0,1]^{2}$ is also $G$ invariant) and point 4) of Proposition 5, for every $\lambda \in \mathbf{R}$,

$$
\begin{aligned}
& \mathbf{E}\left[\exp \left(i \lambda \int_{[0,1]^{2}} \mathbb{U}_{1}\left(t_{1}, t_{2}\right) \mathbb{U}_{2}\left(t_{1}, t_{2}\right) d t_{1} d t_{2}\right)\right] \\
& \quad=\mathbf{E}\left[\exp \left(i \lambda \int_{[0,1]^{2}} \mathbb{U}_{1}^{\pi_{a} \otimes \pi_{a}}\left(t_{1}, t_{2}\right) \mathbb{U}_{2}^{\pi_{a} \otimes \pi_{a}}\left(t_{1}, t_{2}\right) d t_{1} d t_{2}\right)\right]^{4} .
\end{aligned}
$$

3) That is, $\mathbb{W}=\left\{\mathbb{W}_{1}\left(t_{1}, t_{2}\right), \mathbb{W}_{2}\left(t_{1}, t_{2}\right):\left(t_{1}, t_{2}\right) \in[0,1]^{2}\right\}$ is a two-dimensional Gaussian process such that

$$
\begin{aligned}
& \mathbf{E}\left[\mathbb{W}_{1}\left(t_{1}, t_{2}\right), \mathbb{W}_{1}\left(s_{1}, s_{2}\right)\right]=\mathbf{E}\left[\mathbb{W}_{2}\left(t_{1}, t_{2}\right), \mathbb{W}_{2}\left(s_{1}, s_{2}\right)\right]=\left(t_{1} \wedge s_{1}\right)\left(t_{2} \wedge s_{2}\right), \\
& \mathbf{E}\left[\mathbb{W}_{1}\left(t_{1}, t_{2}\right), \mathbb{W}_{2}\left(s_{1}, s_{2}\right)\right]=\mathbf{E}\left[\mathbb{W}_{2}\left(t_{1}, t_{2}\right), \mathbb{W}_{1}\left(s_{1}, s_{2}\right)\right]=\rho\left(t_{1} \wedge s_{1}\right)\left(t_{2} \wedge s_{2}\right) .
\end{aligned}
$$


To conclude, we use point 2) of Proposition 3 to show that

$$
\begin{aligned}
\mathbf{E}\left[\mathbb{U}_{1}^{\pi_{a} \otimes \pi_{a}}\left(t_{1}, t_{2}\right) \mathbb{U}_{1}^{\pi_{a} \otimes \pi_{a}}\left(s_{1}, s_{2}\right)\right] & =\mathbf{E}\left[\mathbb{U}_{2}^{\pi_{a} \otimes \pi_{a}}\left(t_{1}, t_{2}\right) \mathbb{U}_{2}^{\pi_{a} \otimes \pi_{a}}\left(s_{1}, s_{2}\right)\right] \\
& =R_{v}^{\pi_{a} \otimes \pi_{a}}\left(s_{1}, t_{1}\right) R_{v}^{\pi_{a} \otimes \pi_{a}}\left(s_{2}, t_{2}\right), \\
\mathbf{E}\left[\mathbb{U}_{1}^{\pi_{a} \otimes \pi_{a}}\left(t_{1}, t_{2}\right) \mathbb{U}_{2}^{\pi_{a} \otimes \pi_{a}}\left(s_{1}, s_{2}\right)\right] & =\mathbf{E}\left[\mathbb{U}_{2}^{\pi_{a} \otimes \pi_{a}}\left(t_{1}, t_{2}\right) \mathbb{U}_{1}^{\pi_{a} \otimes \pi_{a}}\left(s_{1}, s_{2}\right)\right] \\
& =\rho R_{v}^{\pi_{a} \otimes \pi_{a}}\left(s_{1}, t_{1}\right) R_{v}^{\pi_{a} \otimes \pi_{a}}\left(s_{2}, t_{2}\right),
\end{aligned}
$$

thus implying that

$$
\begin{aligned}
& \left\{\mathbb{U}_{1}\left(t_{1}, t_{2}\right), \mathbb{U}_{2}\left(t_{1}, t_{2}\right):\left(t_{1}, t_{2}\right) \in[0,1 / 2]^{2}\right\} \\
& \quad \stackrel{\text { law }}{=}\left\{4^{-1} \mathbb{B}_{0,1}\left(2 t_{1}, 2 t_{2}\right), 4^{-1} \mathbb{B}_{0,2}\left(2 t_{1}, 2 t_{2}\right):\left(t_{1}, t_{2}\right) \in[0,1 / 2]^{2}\right\},
\end{aligned}
$$

and therefore

$$
\begin{aligned}
\int_{[0,1]^{2}} & \mathbb{U}_{1}^{\pi_{a} \otimes \pi_{a}}\left(t_{1}, t_{2}\right) \mathbb{U}_{2}^{\pi_{a} \otimes \pi_{a}}\left(t_{1}, t_{2}\right) d t_{1} d t_{2} \\
& \stackrel{\text { law }}{=} \frac{1}{4} \int_{[0,1 / 2]^{2}} \mathbb{B}_{0,1}\left(2 t_{1}, 2 t_{2}\right) \mathbb{B}_{0,2}\left(2 t_{1}, 2 t_{2}\right) d t_{1} d t_{2},
\end{aligned}
$$

so that (56) is obtained by a standard change of variables on the right-hand side of the previous expression. The reader is referred to [13] for other twoparameters generalizations of Watson's identity.

4.3. Proof of Theorem 5. 1) Since $G$ is finite, to prove both inequalities in (50) it is sufficient to show that, for every $g, h \in G$,

$$
\int_{Y} \int_{Y} R(h \cdot y, g \cdot z)^{2} \mu(d z) \mu(d y)<+\infty .
$$

But, since $\mu$ is $G$-invariant, and taking into account (45),

$$
\begin{gathered}
\int_{Y} \int_{Y} R(h \cdot y, g \cdot z)^{2} \mu(d z) \mu(d y)=\int_{Y} \int_{Y} R(y, z)^{2} \mu(d z) \mu(d y) \\
=\int_{Y} \int_{Y}\left(\int_{T} \phi_{1}(z, t) \phi_{1}(y, t) \tau(d t)\right)^{2} \mu(d z) \mu(d y)<+\infty
\end{gathered}
$$

due to (49), as well as to an application of the Cauchy-Schwarz inequality.

2) This is a direct consequence of point 1) of Proposition 3. $[\pi] \in \widehat{G}$,

3) By additivity of Gaussian measures, for every $y \in Y, i=1,2$, and

$$
\begin{aligned}
Z_{i}^{\pi}(y) & =\frac{1}{|G|} \sum_{g \in G} Z(g \cdot y) \chi_{\pi}\left(g^{-1}\right)=\frac{1}{|G|} \sum_{g \in G} X\left(\phi_{i}(g \cdot y, \cdot)\right) \chi_{\pi}\left(g^{-1}\right) \\
& =X\left(\phi_{i}^{(\pi)}(y, \cdot)\right)
\end{aligned}
$$


where

$$
\phi_{i}^{(\pi)}(y, t):=\frac{1}{|G|} \sum_{g \in G} \phi_{i}(g \cdot y, t) \chi_{\pi}\left(g^{-1}\right), \quad(y, t) \in Y \times T
$$

(note that $\phi_{i}^{(\pi)} \in L^{2}(d \mu \times d \tau)$ ). Moreover, for any $y_{1}, y_{2} \in Y$,

$$
\begin{aligned}
& \mathbf{E}\left[Z_{1}^{\pi}\left(y_{1}\right) Z_{1}^{\pi}\left(y_{2}\right)\right]=\mathbf{E}\left[Z_{2}^{\pi}\left(y_{1}\right) Z_{2}^{\pi}\left(y_{2}\right)\right]=R^{\pi \otimes \pi}\left(y_{1}, y_{2}\right), \quad \text { and } \\
& \mathbf{E}\left[Z_{1}^{\pi}\left(y_{1}\right) Z_{2}^{\pi}\left(y_{2}\right)\right]=\rho R^{\pi \otimes \pi}\left(y_{1}, y_{2}\right),
\end{aligned}
$$

due to formula (37), thus yielding the desired result.

4) Fix $[\pi] \in \widehat{G}$. Since $Z_{1}^{\pi}$ and $Z_{2}^{\pi}$ are Volterra processes with respect to the Gaussian measure $X$, we may apply a standard version of the multiplication formula for Wiener-Itô integrals (see, e.g., [4, p. 211]) to obtain

$$
\int_{Y} Z_{1}^{\pi}(y) Z_{2}^{\pi}(y) \mu(d y)=\int_{Y} \rho R^{\pi \otimes \pi}(y, y) \mu(d y)+I_{2}^{X}\left(\Phi^{(\pi)}\right),
$$

where $I_{2}^{X}$ stands for a double Wiener-Itô integral with respect to $X$ (see again [4]), and $\Phi^{(\pi)}$ is the symmetrized kernel

$$
\Phi^{(\pi)}\left(t_{1}, t_{2}\right)=\frac{1}{2} \int_{Y}\left[\phi_{1}^{(\pi)}\left(y, t_{1}\right) \phi_{2}^{(\pi)}\left(y, t_{2}\right)+\phi_{1}^{(\pi)}\left(y, t_{2}\right) \phi_{2}^{(\pi)}\left(y, t_{1}\right)\right] \mu(d y),
$$

where $\phi_{i}^{(\pi)}, i=1,2$, is defined as in (57). On the other hand,

$$
\begin{aligned}
\int_{Y} Z_{1}(y) Z_{2}(y) \mu(d y) & =\int_{Y} \rho R(y, y) \mu(d y)+I_{2}^{X}(\Phi), \text { where } \\
\Phi\left(t_{1}, t_{2}\right) & =\frac{1}{2} \int_{Y}\left[\phi_{1}\left(y, t_{1}\right) \phi_{2}\left(y, t_{2}\right)+\phi_{1}\left(y, t_{2}\right) \phi_{2}\left(y, t_{1}\right)\right] \mu(d y) .
\end{aligned}
$$

Now, it is well known that the law of a double Wiener-Itô integral is determined by its cumulants (see [22]). We therefore denote by $\kappa_{n}(J), n \geqslant 1$, the $n$th cumulant of a given random variable $J$, and use a version of the diagram formulae for cumulants of multiple stochastic integrals (as presented, for instance, in [23], [17, Proposition 9 and Corollary 1] or [8, Section 2]) to obtain that, for every $n \geqslant 2$, there exists a universal combinatorial coefficient $c_{n}>0$ such that, for any $[\pi] \in \widehat{G}$,

$$
\kappa_{n}\left(I_{2}^{X}\left(\Phi^{(\pi)}\right)\right)=c_{n} \int_{T^{n}} \Phi^{(\pi)}\left(t_{1}, t_{2}\right) \Phi^{(\pi)}\left(t_{2}, t_{3}\right) \cdots \Phi^{(\pi)}\left(t_{n}, t_{1}\right) \tau\left(d t_{1}\right) \cdots \tau\left(d t_{n}\right)
$$

and also

$$
\kappa_{n}\left(I_{2}^{X}(\Phi)\right)=c_{n} \int_{T^{n}} \Phi\left(t_{1}, t_{2}\right) \Phi\left(t_{2}, t_{3}\right) \cdots \Phi\left(t_{n}, t_{1}\right) \tau\left(d t_{1}\right) \cdots \tau\left(d t_{n}\right) .
$$


By using the relations

$$
\begin{aligned}
& \int_{T} \phi_{1}\left(y_{1}, t\right) \phi_{1}\left(y_{2}, t\right) \tau(d t)=\int_{T} \phi_{2}\left(y_{1}, t\right) \phi_{2}\left(y_{2}, t\right) \tau(d t)=R\left(y_{1}, y_{2}\right), \\
& \int_{T} \phi_{1}\left(y_{1}, t\right) \phi_{2}\left(y_{2}, t\right) \tau(d t)=\int_{T} \phi_{2}\left(y_{1}, t\right) \phi_{1}\left(y_{2}, t\right) \tau(d t)=\rho R\left(y_{1}, y_{2}\right),
\end{aligned}
$$

as well as a combinatorial argument, we finally obtain, for $n \geqslant 2$,

$$
\begin{aligned}
\kappa_{n}\left(I_{2}^{X}\left(\Phi^{(\pi)}\right)\right) & =\frac{c_{n} K(n, \rho)}{2^{n}} \int_{Y}\left[R^{\pi \otimes \pi} \otimes_{(n)} R^{\pi \otimes \pi}\right](y, y) \mu(d y), \\
\kappa_{n}\left(I_{2}^{X}(\Phi)\right) & =\frac{c_{n} K(n, \rho)}{2^{n}} \int_{Y}\left[R \otimes_{(n)} R\right](y, y) \mu(d y) .
\end{aligned}
$$

To conclude, use independence to write, for $n \geqslant 1$,

$$
\kappa_{n}\left(\int_{Y} Z_{1}(y) Z_{2}(y) \mu(d y)\right)=\sum_{[\pi] \in \widehat{G}} \kappa_{n}\left(\int_{Y} Z_{1}^{\pi}(y) Z_{2}^{\pi}(y) \mu(d y)\right),
$$

and observe that, thanks to the translation invariance property of cumulants (see, e.g., [16, Corollary 4.1]), for any $n \geqslant 2$,

$$
\begin{aligned}
\kappa_{n}\left(\int_{Y} Z_{1}(y) Z_{2}(y) \mu(d y)\right) & =\kappa_{n}\left(I_{2}^{X}(\Phi)\right), \\
\kappa_{n}\left(\int_{Y} Z_{1}^{\pi}(y) Z_{2}^{\pi}(y) \mu(d y)\right) & =\kappa_{n}\left(I_{2}^{X}\left(\Phi^{(\pi)}\right)\right), \quad[\pi] \in \widehat{G},
\end{aligned}
$$

and consequently

$$
\kappa_{n}\left(I_{2}^{X}(\Phi)\right)=\sum_{[\pi] \in \widehat{G}} \kappa_{n}\left(I_{2}^{X}\left(\Phi^{(\pi)}\right)\right) .
$$

The proof is completed by standard arguments.

\section{Refinements and further applications.}

5.1. Connections with Karhunen-Loève expansions. In this section, we elucidate some of the connections between our decomposition of stochastic processes, and Karhunen-Loève (KL) expansions of Gaussian processes indexed by the elements of a measurable space $(T, \mathscr{T})$ (for fundamental facts about KL expansions, see, e.g., [1], [21, Chap. 5], as well as [3] and the references therein). In what follows, $G$ is a topological compact group, acting on $T$ through the application $(g, t) \mapsto g \cdot t, t \in T$. We write $m(d t)$ to indicate a $G$-invariant measure on $(T, \mathscr{T})$. We also consider a positive definite kernel $R(s, t), s, t \in T$, such that $R$ is the covariance function of a real-valued, centered Gaussian process $\mathbb{X}=\{\mathbb{X}(t): t \in T\}$, defined on some probability space $(\Omega, \mathscr{F}, \mathbf{P})$, and such that, for every $\omega \in \Omega$, the function $t \mapsto \mathbb{X}(t)$ is in $L^{2}(T, d m)$. We denote by $\lambda_{1}>\lambda_{2}>\cdots>0$ the sequence of the eigenvalues of $R$ (with respect to $m(\cdot)$ ), whereas $E_{1}, E_{2}, \ldots$ indicate the 
associated eigenspaces. For every $j \geqslant 1, n_{j}$ is the (finite) dimension of $E_{j}$. The next assumption, ensuring that $\mathbb{X}$ can be represented (in some weak sense) as a KL series, will be in order throughout the section.

A s s u m ption C. For every $j$, denote by $\left(f_{j, 1}, \ldots, f_{j, n_{j}}\right)$ an orthonormal basis of $E_{j}$ (with respect to the inner product of the space $\left.L^{2}(T, d m)\right)$.

(C-i) The process $\mathbb{X}$ admits the following KL expansion: there exists an array of i.i.d. $N(0,1)$ random variables $\left\{\xi_{j, l}: j \geqslant 1, l=1, \ldots, n_{j}\right\}$ such that, as $N \rightarrow+\infty$ and $\mathbf{P}$-a.s., the process

$\mathbb{X}_{N}(t)=\sum_{j=1}^{N} \sqrt{\lambda_{j}}\left\{\xi_{j, 1} \times f_{j, 1}(t)+\xi_{j, 2} \times f_{j, 2}(t)+\cdots+\xi_{j, n_{j}} \times f_{j, n_{j}}(t)\right\}, \quad t \in T$,

converges to $\mathbb{X}$ in $L^{2}(T, d m)$.

(C-ii) The processes $\mathbb{X}, \mathbb{X}_{N}(N \geqslant 1)$ and $f_{j, l}\left(j \geqslant 1, l=1, \ldots, n_{j}\right)$ satisfy Assumption A of Section 3.1, with $Y=T$.

The reader is referred once again to [1] or [21] for (rather general) sufficient conditions, ensuring the validity of Assumption (C-i) in the case $[0,1]^{d}$, $d \geqslant 1$. Assumption (C-ii) is redundant for $G$ finite.

According to (12), for every $[\pi] \in \widehat{G}$ and every $N \geqslant 1$, we define

$$
\begin{aligned}
f^{\pi}(t) & =d_{\pi} \int_{G} \chi_{\pi}(g) f\left(g^{-1} \cdot t\right) d g, \quad f \in L^{2}(T, d m), \\
\mathbb{X}_{N}^{\pi}(t) & =d_{\pi} \int_{G} \chi_{\pi}(g) \mathbb{X}_{N}\left(g^{-1} \cdot t\right) d g \\
& =\sum_{j=1}^{N} \sqrt{\lambda_{j}}\left\{\xi_{j, 1} \times f_{j, 1}^{\pi}(t)+\cdots+\xi_{j, n_{j}} \times f_{j, n_{j}}^{\pi}(t)\right\}, \\
\mathbb{X}^{\pi}(t) & =d_{\pi} \int_{G} \chi_{\pi}(g) \mathbb{X}\left(g^{-1} \cdot t\right) d g .
\end{aligned}
$$

Note that, according to point 3) of Proposition 3, P-a.s.,

$$
\mathbb{X}(t)=\sum_{[\pi] \in \widehat{G}} \mathbb{X}^{\pi}(t)
$$

with convergence in $L^{2}(T, d m)$. The following proposition states some remarkable relations between (59) and KL expansions, in the case of Gaussian processes with a $G$-invariant law. Note that, given an irreducible representation $\pi$, we denote by $\bar{\pi}$ the irreducible representation defined by the homomorphism $g \mapsto \bar{\pi}(g), g \in G$, where $\bar{\pi}(g)$ is the matrix whose entries are the complex conjugate of the entries of $\pi(g)$ (this is also known as the contragradient representation associated with $\pi$, see, e.g., the discussion contained in $[7$, Chap. 4]). 
Proposition 6. Let the notation and assumptions of this section prevail. Then,

1) for each $[\pi] \in \widehat{G}, \mathbb{X}_{N}^{\pi}(t) \rightarrow \mathbb{X}^{\pi}(t)$ as $N \rightarrow+\infty, \mathbf{P}$-a.s. in $L^{2}(T, d m)$;

2 ) suppose $\mathbb{X}$ has a $G$-invariant law; then, for each $j \geqslant 1$, the application $g \mapsto\left\{f(t) \mapsto f\left(g^{-1} \cdot t\right): f \in E_{j}\right\}$ is a finite dimensional representation of $G$;

3) for $j \geqslant 1$, write $E_{j}=E_{j}^{1} \oplus \cdots \oplus E_{j}^{h_{j}}$, with $1 \leqslant h_{j} \leqslant n_{j}$, to indicate the canonical decomposition of $E_{j}$, where $E_{j}^{l}\left(l=1, \ldots, h_{j}\right)$ is the direct sum of the irreducible representations contained in $E_{j}$ that are equivalent to the same $\left[\pi_{j, l}\right] \in \widehat{G}$ (see $\left[19\right.$, Section 2.6]); then, for every $\pi \in \widehat{G}, f^{\pi}$ (as defined in (58)) is equal to zero if $\left[\pi_{j, l}\right] \neq[\bar{\pi}]$ for every $l=1, \ldots, h_{j}$, and equal to the projection of $f$ on $E_{j}^{l}$ if $\left[\pi_{j, l}\right]=[\bar{\pi}]$ for some $l=1, \ldots, h_{j}$.

P r o o f. 1) Just write

$$
\begin{aligned}
\int_{T}\left(\mathbb{X}_{N}^{\pi}(t)-\mathbb{X}^{\pi}(t)\right)^{2} m(d t) \\
\quad=d_{\pi}^{2} \int_{T}\left(\int_{G} \chi_{\pi}(g)\left(\mathbb{X}_{N}\left(g^{-1} \cdot t\right)-\mathbb{X}\left(g^{-1} \cdot t\right)\right) d g\right)^{2} m(d t) \\
\quad \leqslant d_{\pi}^{2} \alpha_{\pi}^{2} \int_{T}\left[\int_{G} \chi_{\pi}(g)\left(\mathbb{X}_{N}\left(g^{-1} \cdot t\right)-\mathbb{X}\left(g^{-1} \cdot t\right)\right)^{2} d g\right] m(d t) \\
\quad=d_{\pi}^{2} \alpha_{\pi}^{2} \int_{T}\left(\mathbb{X}_{N}(t)-\mathbb{X}(t)\right)^{2} m(d t) \rightarrow 0,
\end{aligned}
$$

thanks to Assumption $\mathrm{C}$, as well as the $G$-invariance of $m$.

2) A function $f$ is in $E_{j}$ if and only if $\lambda_{j} f(t)=\int_{T} R(t, s) f(s) m(d s)$. Now suppose $\mathbb{X}$ has a $G$-invariant law. Then, $R$ is also $G$-invariant, and moreover, for every $g \in G$ and $f \in E_{j}$,

$$
\begin{aligned}
f\left(g^{-1} \cdot t\right) & =\int_{T} R\left(g^{-1} \cdot t, s\right) f(s) m(d s) \\
& =\int_{T} R\left(g^{-1} \cdot t, g^{-1} \cdot s\right) f\left(g^{-1} \cdot s\right) m(d s) \quad(G \text {-invariance of } m) \\
& =\int_{T} R(t, s) f\left(g^{-1} \cdot s\right) m(d s) \quad(G \text {-invariance of } R),
\end{aligned}
$$

and therefore $f \in E_{j}$. This concludes the proof.

3) This point is a direct application of Theorem 8 in [19].

5.2. Watson's identity on the $n$-dimensional flat tori. Watson's identity concerns processes defined on $[0,1]$ and taking the same values at $t=0$ and $t=1$, in other words on a circle. Among the various geometrical sets arising as generalizations of the circle in higher dimensions, we will consider the $n$-dimensional torus. Recall that an $n$-dimensional lattice is a set

$$
\Gamma:=\left\{\sum_{i=1}^{n} a_{i} v_{i}: a_{1}, \ldots, a_{n} \in \mathbf{Z}\right\}
$$


where $v_{1}, \ldots, v_{n}$ are $n$ independent vectors in $\mathbf{R}^{n}$. The dual lattice $\Gamma^{*}$ is defined to be the set of $v^{*} \in \mathbf{R}^{n}$ such that $\left\langle v \mid v^{*}\right\rangle \in \mathbf{Z}$, for all $v \in \Gamma$. The quotient space $T_{\Gamma}:=\mathbf{R}^{n} / \Gamma$ is the $n$-dimensional torus associated with $\Gamma$, and it is endowed with the measure $d m$ inherited from the Lebesgue measure on $\mathbf{R}^{n}$. Consider a centered Gaussian process $X:=\left\{X(t): t \in T_{\Gamma}\right\}$, with covariance function $K$. For $n=1, \Gamma=\mathbf{Z}$, one has $T^{1}=\mathbf{R} / \mathbf{Z}$ and $X$ is a centered Gaussian process defined on $[0,1]$ such that $X(0)=X(1)$. In this case $X$ can be a Brownian bridge or the Watson process. These processes are involved in Watson's identity (1). We propose an assumption on $X$, implying that this process satisfies an identity analogue of Watson's duplication identity (1) in higher dimensions. The techniques we adopt represent a $n$-dimensional generalization of the line of reasoning that the second author used in [14].

A s s u m ption D. There exists a function $k: T_{\Gamma} \rightarrow \mathbf{R}$ such that

$$
K(s, t)=k(t-s) \quad\left(s, t \in T_{\Gamma}\right) .
$$

Note that this assumption is equivalent to the hypothesis that $K$ is invariant under the isometry group of $T_{\Gamma}$ which is composed of all translations of vector $v \in\left\{\sum_{i=1}^{n} a_{i} v_{i}: 0 \leqslant a_{i} \leqslant 1,1 \leqslant i \leqslant n\right\}$. Let us first check that the covariance function of Watson's process given by (55) can be put in the form (60). If for $s \in \mathbf{R}$ we denote by $\bar{s} \in[0,1)$ the corresponding class in $\mathbf{R} / \mathbf{Z}$, we have, for $s, t \in[0,1]$,

$$
|s-t|-\frac{1}{2}= \begin{cases}t-s-\frac{1}{2}=\frac{1}{2}-(1+s-t)=\frac{1}{2}-\overline{(s-t)} & \text { if } s<t, \\ s-t-\frac{1}{2}=\overline{(s-t)}-\frac{1}{2} & \text { if } s \geqslant t\end{cases}
$$

hence

$$
|s-t|-\frac{1}{2}= \pm\left(\overline{(s-t)}-\frac{1}{2}\right) \quad(s, t \in[0,1]) .
$$

This allows us to obtain the expression

$$
\begin{aligned}
s \wedge t-\frac{s+t}{2}+\frac{(s-t)^{2}}{2}+\frac{1}{12} & =\frac{1}{2}\left(|s-t|-\frac{1}{2}\right)^{2}-\frac{1}{24} \\
& =\frac{1}{2}\left(\overline{(s-t)}-\frac{1}{2}\right)^{2}-\frac{1}{24}=: k(u)
\end{aligned}
$$

where $k(u)=(\bar{u}-1 / 2)^{2} / 2-1 / 24$ for $u \in T^{1}$.

Lemma 1. If the centered Gaussian process $X$ satisfies Assumption D, then $K$ admits a Karhunen-Loève expansion of the form

$K(s, t)=\sum_{v \in \Gamma^{*}} \lambda_{v}\left\{\alpha_{v} \cos (2 \pi\langle v \mid s\rangle) \cos (2 \pi\langle v \mid t\rangle)+\alpha_{v} \sin (2 \pi\langle v \mid s\rangle) \sin (2 \pi\langle v \mid t\rangle)\right\}$, 
where $\lambda_{v} \in[0, \infty)$ for each $v \in \Gamma^{*}$, and $\alpha_{v}>0$ is chosen such that

$$
\int_{T_{\Gamma}} \alpha_{v}^{2} \cos ^{2}(2 \pi\langle v \mid s\rangle) d m(s)=\int_{T_{\Gamma}} \alpha_{v}^{2} \sin ^{2}(2 \pi\langle v \mid s\rangle) d m(s)=1 .
$$

P r o o f. The functions $\left\{u \mapsto \cos (2 \pi\langle v \mid u\rangle), u \mapsto \sin (2 \pi\langle v \mid u\rangle): v \in \Gamma^{*}\right\}$ form a complete set of orthogonal functions in $L^{2}\left(T_{\Gamma}\right)$. The Fourier series of $k$ in this basis has the form

$$
k(u)=\sum_{v \in \Gamma^{*}}\left\{a_{v} \cos (2 \pi\langle v \mid u\rangle)+b_{v} \sin (2 \pi\langle v \mid u\rangle)\right\} .
$$

Since $k(u)=k(x-y)=K(x, y)=K(y, x)=k(y-x)=k(-u)$, one has $b_{v}=0$ for each $v \in \Gamma^{*}$. If we replace $u$ by $x-y$ and use the identity $\cos (a-b)=\cos a \cos b+\sin a \sin b$ we obtain the desired Karhunen-Loève expansion of $K$.

Theorem 6. If the centered Gaussian process $X$ satisfies Assumption $\mathrm{D}$, then

$$
\int_{T} X^{2}(t) d m(t)=\frac{1}{4} \int_{T} X_{1}^{2}(t) d m(t)+\frac{1}{4} \int_{T} X_{2}^{2}(t) d m(t),
$$

where $X_{1}(t):=(X(t)-X(-t)) / 2$ and $X_{2}(t):=(X(t)+X(-t)) / 2$ are two independent centered Gaussian processes such that

$$
\int_{T} X_{1}^{2}(t) d m(t) \stackrel{\text { law }}{=} \int_{T} X_{2}^{2}(t) d m(t)
$$

and $X_{1}(0, \ldots, 0)=X_{1}\left(v_{1} / 2, \ldots, v_{n} / 2\right)=0$.

P r o o f. From the preceding lemma, $X$ has a Karhunen-Loève expansion of the form

$$
X(t)=\sum_{v \in \Gamma^{*}} \lambda_{v}\left\{\xi_{v} \alpha_{v} \cos (2 \pi\langle v \mid t\rangle)+\xi_{v}^{\prime} \alpha_{v} \sin (2 \pi\langle v \mid t\rangle)\right\}
$$

(where the $\xi_{v}$ and the $\xi_{v}^{\prime}$ are independent standard Gaussian random variables) and the claimed identity is clearly fulfilled with

$$
X_{1}(t)=\sum_{v \in \Gamma^{*}} \lambda_{v} \xi_{v} \alpha_{v} \sin (2 \pi\langle v \mid t\rangle), \quad X_{2}(t)=\sum_{v \in \Gamma^{*}} \lambda_{v} \xi_{v} \alpha_{v} \sin (2 \pi\langle v \mid t\rangle) .
$$

\section{СПИСОК ЛИТЕРАТУРЫ}

1. Adler R. J. An Introduction to Continuity, Extrema, and Related Topics for General Gaussian Processes. Hayward: Inst. Math. Statist., 1990, 160 p. (IMS Lecture Notes Monogr. Ser., v. 12.)

2. Chou C.-S. Sur l'extension d'une identité en loi entre le pont brownien et la variance du mouvement brownien. - Stochastic Process. Appl., 1994, v. 49, № 2, p. 217-225. 
3. Deheuvels P., Peccati G., Yor M. On quadratic functionals of the Brownian sheet and related processes. - Stochastic Process. Appl., 2006, v. 116, № 2, p. 493-538.

4. Dellacherie C., Maisonneuve B., Meyer P.-A. Probabilités et Potentiel (Chapitres XVII à XXIV). Paris: Hermann, 1992.

5. Diaconis P. Group Representations in Probability and Statistics. Hayward: Inst. Math. Statist., 1988, 198 p. (IMS Lecture Notes Monogr. Ser., v. 11.)

6. Dudley R. Real Analysis and Probability. Cambridge: Cambridge Univ. Press, 2002, $555 \mathrm{p}$.

7. Duistermaat J. J., Kolk J. A. C. Lie Groups. Berlin: Springer-Verlag, 1997, 344 p.

8. Fox R., Taqqu M. Multiple stochastic integrals with dependent integrators. - J. Multivariate Anal., 1987, v. 21, № 1, p. 105-127.

9. James G. D., Liebeck M. Representations and Characters of Groups. New York: Cambridge Univ. Press, 2001, 458 p.

10. Marinucci D., Peccati G. Group representations and high-resolution central limit theorems for subordinated spherical random fields. Preprint, 2008.

11. Marinucci D., Peccati G. High-frequency asymptotics for subordinated stationary fields on an Abelian compact group. - Stochastic Process. Appl., 2008, v. 118, № 4, p. $585-613$.

12. Marinucci D., Peccati G. Representations of $S O(3)$ and angular polyspectra. Preprint, 2008.

13. Peccati G., Yor M. Identities in law between quadratic functionals of bivariate Gaussian processes, through Fubini theorems and symmetric projections. Approximation and Probability. Warsaw: Polish Acad. Sci., 2006, p. 235-250. (Banach Center Publ., v. 72.)

14. Pycke J.-R. Sur une identité en loi entre deux fonctionnelles quadratiques du pont Brownien. - C. R. Math. Acad. Sci. Paris, 2005, v. 340, № 5, p. 373-376.

15. Pycke J.-R. A decomposition for invariant tests of uniformity on the sphere. - Proc. Amer. Math. Soc., 2007, v. 135, № 9, p. 2983-2993.

16. Rota G.-C., Shen J. On the combinatorics of cumulants. - J. Combin. Theory Ser. A, 2000, v. 91, № 1-2, p. 283-304.

17. Rota G.-C., Wallstrom T. C. Stochastic integrals: a combinatorial approach. - Ann. Probab., 1997, v. 25, № 3, p. 1257-1283.

18. Serfling R. J. Approximation Theorems of Mathematical Statistics. New York: Wiley, 1980, $371 \mathrm{p}$.

19. Serre J.-P. Linear Representations of Finite Groups. New York-Heidelberg: SpringerVerlag, 1977, 170 p. (Grad. Texts in Math., v. 42.)

20. Shi Z., Yor M. On an identity in law for the variance of the Brownian bridge. - Bull. London Math. Soc., 1997, v. 29, № 1, p. 103-108.

21. Shorack G. R., Wellner J. A. Empirical Processes with Applications to Statistics. New York.: Wiley, 1986, $938 \mathrm{p}$

22. Slud E. $V$. The moment problem for polynomial forms in normal random variables. Ann. Probab., v. 21, № 4, p. 2200-2214.

23. Surgailis D. CLTs for polynomials of linear sequences: Diagram formula with illustrations. Theory and Applications of Long-Range Dependence. Ed. by P. Doukhan et al. Boston: Birkhäuser, 2003, p. 111-127.

24. Watson G. S. Goodness-of-fit tests on a circle. - Biometrika, 1961, v. 48, p. 109-114.

Поступила в редакцию

14.III.2007

Исправленный вариант

16.X.2008 